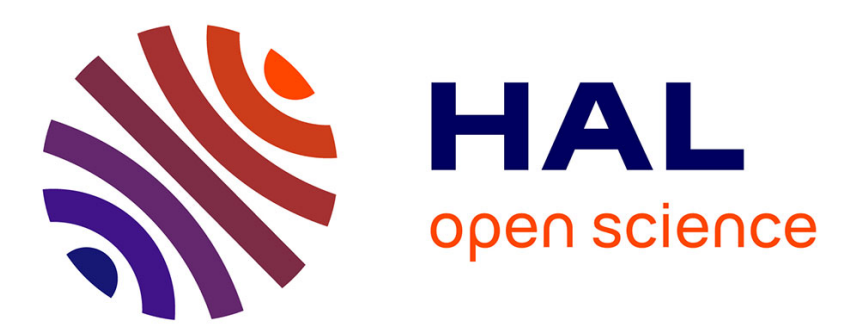

\title{
Single crystal anisotropy and coupled stability analysis for variant reorientation in Magnetic Shape Memory
} Alloys

Krishnendu Haldar, Georges Chatzigeorgiou, Dimitris C. Lagoudas

\section{- To cite this version:}

Krishnendu Haldar, Georges Chatzigeorgiou, Dimitris C. Lagoudas. Single crystal anisotropy and coupled stability analysis for variant reorientation in Magnetic Shape Memory Alloys. European Journal of Mechanics - A/Solids, 2015, 54, pp.53-73. 10.1016/j.euromechsol.2015.06.005 . hal-01199907

\section{HAL Id: hal-01199907 \\ https://hal.science/hal-01199907}

Submitted on 16 Sep 2015

HAL is a multi-disciplinary open access archive for the deposit and dissemination of scientific research documents, whether they are published or not. The documents may come from teaching and research institutions in France or abroad, or from public or private research centers.
L'archive ouverte pluridisciplinaire HAL, est destinée au dépôt et à la diffusion de documents scientifiques de niveau recherche, publiés ou non, émanant des établissements d'enseignement et de recherche français ou étrangers, des laboratoires publics ou privés. 


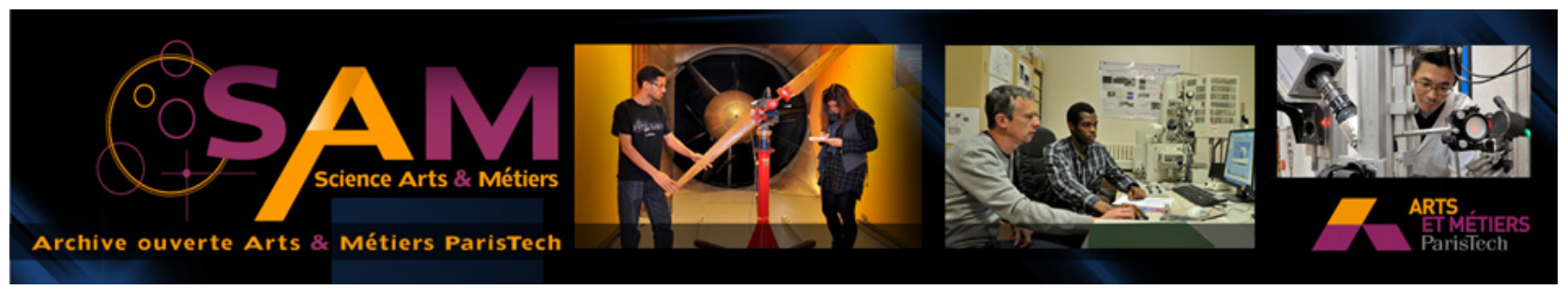

\section{Science Arts \& Métiers (SAM)}

is an open access repository that collects the work of Arts et Métiers ParisTech researchers and makes it freely available over the web where possible.

This is an author-deposited version published in: http://sam.ensam.eu

Handle ID: .http://hdl.handle.net/10985/10024

\section{To cite this version :}

Krishnendu HALDAR, George CHATZIGEORGIOU, Dimitris C. LAGOUDAS - Single crystal anisotropy and coupled stability analysis for variant reorientation in Magnetic Shape Memory Alloys - European Journal of Mechanics A/Solids - Vol. 54, p.53-73 - 2015 


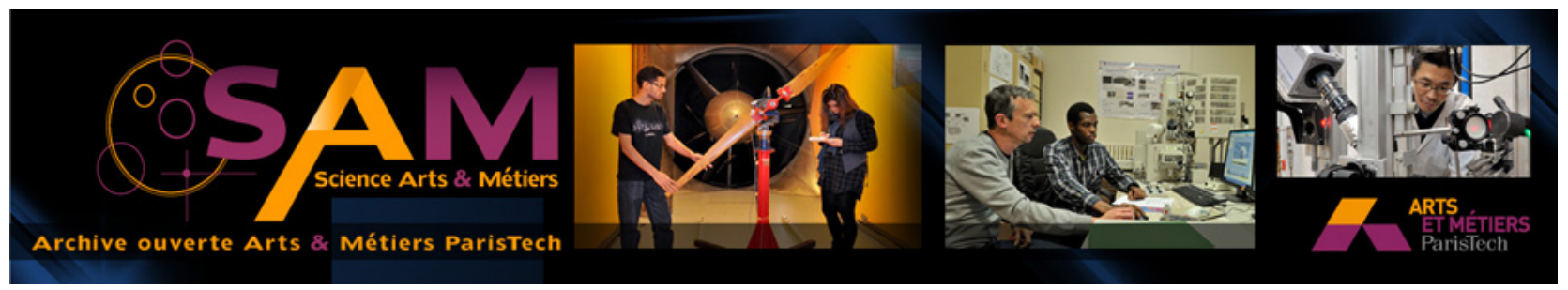

Science Arts \& Métiers (SAM)

is an open access repository that collects the work of Arts et Métiers ParisTech researchers and makes it freely available over the web where possible.

This is an author-deposited version published in: http://sam.ensam.eu Handle ID: .http://hdl.handle.net/null

\section{To cite this version :}

Krishnendu HALDAR, George CHATZIGEORGIOU, Dimitris C. LAGOUDAS - Single crystal anisotropy and coupled stability analysis for variant reorientation in Magnetic Shape Memory Alloys - European Journal of Mechanics A/Solids - Vol. 54, p.53-73 - 2015 


\title{
Single crystal anisotropy and coupled stability analysis for variant reorientation in Magnetic Shape Memory Alloys
}

\author{
Krishnendu Haldar ${ }^{\text {a }}$, George Chatzigeorgiou ${ }^{c}$, Dimitris C. Lagoudas a, b, * \\ a Department of Aerospace Engineering, Texas A\&M University, College Station, TX 77843, USA \\ b Department of Materials Science and Engineering, Texas A\&M University, College Station, TX 77843, USA \\ ${ }^{c}$ LEM3, UMR 7239, Arts et Mtiers ParisTech, Metz-Lorraine, 57078 Metz, France
}

\begin{abstract}
A B S T R A C T
Magnetic Shape Memory Alloys (MSMAs) have been the subject of much research in recent years as potential high-actuation-energy multifunctional materials. In this work we analyze coupled magnetomechanical stability analysis of a variant reorientation mechanism for a single crystal based on a proposed 3-D magneto-mechanically coupled constitutive equations, derived in a consistent thermodynamic way. Discrete symmetry is considered to take into account single crystal anisotropy in the modeling. Analytical results are presented to demonstrate the influence of coupling and anisotropy in the stability of such a material system. Finally, a coupled Boundary Value Problem (BVP) using finite element analysis is performed by considering actual specimen geometry and boundary conditions that are used in the experiments. The numerical simulation reveals localization zones in the solutions due to the loss of ellipticity of the coupled magneto-mechanical problem.
\end{abstract}

\section{Introduction}

MSMAs are best known for their unique ability to produce Magnetic Field Induced Strains (MFIS) up to 10\% under a magnetic field (O'Handley, 1998; O'Handley et al., 2000; Müllner et al., 2003; Shield, 2003). Some of the commonly used MSMA material systems are NiMnGa (Murray et al., 2001a; O'Handley et al., 2003; Heczko et al., 2003; Likhachev et al., 2004), FePd (James and Wuttig, 1998; Yamamoto et al., 2004) and NiMnX, where $X=$ In,Sn,Sb (Sutoua et al., 2004). The unique magneto-mechanical coupling makes MSMAs promising materials for multifunctional structures, actuator and sensor applications (Pasquale, 2003; Tellinen et al., 2002; Sarawate and Dapino, 2006, 2007; Karaman et al., 2007).

The coupled MSMA behaviors can be modeled by considering the material as an electromagnetic continuum. Extensive work on different electromagnetic formulations had been proposed in the literature (Toupin, 1956, 1960; Penfield and Haus, 1967; Hutter et al., 2006; Eringen and Maugin, 1990) based on different notion of breaking up long range and short range forces. In a recent work,
(DeSimone, 1993; DeSimone and Podio-Guidugli, 1996) proposed a continuum theory for deformable ferromagnetic materials. Dorfmann and Ogden (2004, 2005) derived a theory of nonlinear magneto-elasticity for magneto sensitive elastomers. McMeeking and Landis (2005), McMeeking et al. (2007) presented a study of electrostatic forces on large deformations of polarizable materials. A theory for the equilibrium response of magneto-elastic membranes is formulated by Steigmann (2004, 2009). Podio-Guidugli et al. (2010) formulated a continuum theory for the evolution of magnetization and temperature in a rigid magnetic body for ferro/ paramagnetic transition. The variational formulations for general magneto-mechanical materials have been proposed by many authors Kankanala and Triantafyllidis (2004), Ericksen (2006), Bustamante et al. (2008), Miehe et al. (2011b,a).

The macroscopically observable MFIS in MSMAs is caused by the microstructural reorientation of martensitic variants (O'Handley et al., 2000; Karaca et al., 2006), field induced phase transformation (Sutoua et al., 2004; Kainuma et al., 2006; Karaca et al., 2007,2009 ) or a combination of the two mechanisms. In this work we will focus on variant reorientation. In the variant reorientation mechanism, the variants have different preferred directions of magnetization and the magnetic field is applied to select certain variants among others, which results in the macroscopic shape change. 
There are two major modeling approaches for variant reorientation mechanism. In microstructural based models, the resulting macroscopic strain and magnetization response are predicted by minimizing a free energy functional. Details on the microstructural based modeling approach can be found in (James and Wuttig, 1998; DeSimone and James, 1997; O'Handley, 1998; Murray et al., 2001b; DeSimone and James, 2002, 2003). The second approach to study the material behavior is through thermodynamics based phenomenological modeling (Hirsinger and Lexcellent, 2003b, a; Kiefer and Lagoudas, 2005; Kiefer et al., 2006; Kiefer and Lagoudas, 2009). Most recent development of a variational modeling of variant reorientation in MSMAs can be found in Wang and Steinmann (2012). Modeling of field induced phase transformation with detailed experimental characterization can be found in Haldar et al. (2014). The effects of magnetic body force and couple on the variant reorientation mechanism are investigated through a coupled boundary value problem in Haldar et al. (2011).

One of the major challenges for understanding the magnetostatic response of the MSMAs is the experimental measurement of the magnetic field inside the material. The measurements of the magnetic field are strongly influenced by the shape and size of the specimens Shield (2003). In general, the shape of the specimen, used in the experiments, is a prismatic bar with a rectangular cross section. A compressive stress is applied along the axis of the prismatic bar and a magnetic field is applied along the perpendicular direction of the axis Shield (2003), Kiefer et al. (2006). In order to build a reliable constitutive model, one needs to relate the measured data of the applied magnetic field with the magnetic field in the material. A numerical technique to correct the constitutive responses from the demagnetization effect is also described in Haldar et al. (2011).

During the reorientation process, the nonuniformity caused by the shape effect combined with the strong nonlinear constitutive response in magnetization leads to localization of the numerical solution. We investigated this phenomenon in Haldar et al. (2010), where we found that the magnetic field during reorientation changes drastically in the band like localized zones.

In this study, there are two major contributions. The first is that the considered constitutive equation includes single crystal anisotropy. A rate form for the mechanical and magnetization constitutive equations is derived to facilitate the stability analysis in an efficient way. We analytically perform stability analysis for magneto-mechanical coupling with anisotropy, where our previous work was confined only within magnetostatic analysis with isotropic assumption. We found that a material parameter due to anisotropy indeed influences the stability of the system. The second major point is the implementation of the coupled magnetomechanical stability analysis. In the FE analysis, our main effort is to understand how instability caused by the magnetic behavior affects the mechanical field variables. So, we only considered isotropy in the FE analysis to solve a relatively simple problem. Additional efforts are necessary to see the influences of anisotropy in the numerical analysis, which are not considered in this study.

The organization of the paper is as follows: In Section 2, we briefly introduce a thermodynamic based continuum framework for a coupled magneto-mechanical dissipative system. A specific form of the Gibbs free energy is proposed in Section 3 and the constitutive equations are derived with discrete symmetry restrictions. An incremental form of the constitutive equations with magnetic and mechanical tangent stiffness matrices is presented in 4 and the stability analysis of such a coupled system is presented in Section 5. Some numerical examples are presented in Section 6. Finally, in Section 7 we solved a boundary value problem to demonstrate the appearance of band-like structure due to loss of ellipticity. Parameter identifications and validation of the newly proposed constitutive equations are essential. As it is not our main focus, we present a concise but detailed descriptions in the B.

\section{Continuum approach for single crystal MSMA modeling}

In this section we briefly outline the thermodynamic framework to describe the constitutive equations. Evolution equations of the internal state variables, introduced to capture the hysteretic behavior of the magneto-mechanical response, are also discussed.

\subsection{Thermodynamic framework}

We denote the reference configuration by $\Omega_{0}$, which is free from any externally applied stimuli and the current configuration by $\Omega$. The body consists of material points $\mathbf{X} \in \Omega_{0}$. The spatial position in the deformed configuration is denoted by $\mathbf{x}=\varphi(\mathbf{X}, t)$ and the deformation gradient is defined by $\mathbf{F}=\nabla_{\mathbf{X}} \varphi$ with $J=\operatorname{det} F>0$. In the deformed configuration $\Omega$, we denote the magnetic induction by $\mathbf{b}$, the magnetic field by $\mathbf{h}$ and the magnetization vector by $\mathbf{m}$. The free current (conductive) density of the body is neglected in this study.

The constitutive response of MSMAs undergoing variant reorientation will depend on state variables such as appropriate measures of stress and magnetic field and also internal state variables to account for loading path dependence due to the hysteretic response caused by dissipation. Using the Coleman and Noll procedure (Coleman and Noll, 1963), the following constitutive equations can be obtained

$\mathbf{E}=-\rho_{0} G, \mathbf{s}^{E}$,

$\mu_{0} \mathbf{M}=-\rho_{0} G, \mathbf{H}$

$S=-G,_{T}$

$-\rho G, \mathbb{Z}_{i} \cdot \dot{\mathbb{Z}}_{i} \geqslant 0$.

Here $\mathbf{E}=\frac{1}{2}(\mathbf{C}-\mathbf{I}), \mathbf{C}=\mathbf{F}^{\mathbf{T}} \mathbf{F}, \rho_{0}$ is the density, $\mathbf{M}=J \mathbf{F}^{-1} \mathbf{m}$ is the magnetization, $\mathbf{H}=\mathbf{F}^{T} \mathbf{h}$ is the magnetic field and $\{\mathbb{Z}\}$ is the set of internal variables in $\Omega_{0} \cdot G\left(\mathbf{S}^{E}, \mathbf{H}, T,\{\mathbb{Z}\}\right)$ is the Gibbs free energy, $\mathbf{S}^{E}=J \mathbf{F}^{-1} \boldsymbol{\sigma}^{E} \mathbf{F}^{-T}$, where $\boldsymbol{\sigma}^{E}=\boldsymbol{\sigma}-\boldsymbol{\sigma}^{M}, \boldsymbol{\sigma}$ is the total stress (Toupin, 1956, 1960) generated due to combined magneto-mechanical effect (i.e. if $\mathbf{t}$ is the total traction, then $\mathbf{t}=\boldsymbol{\sigma n}), \boldsymbol{\sigma}^{M}$ is the Maxwell stress in the deformed configuration and $T$ is the temperature. On deriving Eq. (1), one needs to start from the internal energy and introduce a proper Legendre transformation with regard to the deformation gradient $\mathbf{F}$ and magnetic induction $\mathbf{b}$ in the deformed configuration. Detailed derivations can be found in (Haldar and Lagoudas, 2014; Haldar, 2012). Our next step is to define the set of internal variables $\{\mathbb{Z}\}$.

\subsubsection{Internal state variables}

We assume that the volume fraction of the newly oriented field induced variant is $\xi$. We further consider that the inelastic strain $\mathbf{E}^{r}$ and the internal magnetization $\mathbf{M}^{r}$, generated during variant reorientation, are tensorial internal variables of order two and one respectively. The internal magnetization takes into account the phenomenological effect of different micro-magnetic mechanisms like the rotation of magnetization vector and the evolution of magnetic domain walls. Finally, we consider the mixing energy $g^{r}$ of the reorientation as an internal variable. So the complete list of the internal state variables is $\{\mathbb{Z}\}=\left\{\mathbf{E}^{r}, \mathbf{M}^{r}, \xi, g^{r}\right\}$. Expanding the entropy inequality (1d), we obtain 
$\boldsymbol{\pi}_{\mathbf{E}^{r}}: \dot{\mathbf{E}}^{r}+\boldsymbol{\pi}_{\mathbf{M}^{r}} \cdot \dot{\mathbf{M}}^{r}+\pi_{\xi} \dot{\xi}+\pi_{g^{r}} g^{r} \geq 0$.

The thermodynamic driving forces are denoted by

$$
\begin{aligned}
& \boldsymbol{\pi}_{\mathbf{E}^{r}}=-\rho G, \mathbf{E}^{r}, \\
& \boldsymbol{\pi}_{\mathbf{M}^{r}}=-\rho G, \mathbf{M}^{r}, \\
& \pi_{\xi}=-\rho G, \xi \\
& \pi_{g^{r}}=-\rho G, g^{r} .
\end{aligned}
$$

We further assume that the reorientation strain rates obey the following flow rules

$$
\dot{\mathbf{E}}^{r}=\Lambda^{r} \dot{\xi}
$$

The $\Lambda^{r}$ is the tensor which takes into account the direction and magnitude of the generated strain during variant reorientation. Similarly we consider that the rate of magnetization vector generated during reorientation follows the following flow rules

$$
\dot{\mathbf{M}}^{r}=\boldsymbol{\gamma}^{r} \dot{\xi}
$$

where $\gamma^{r}$ takes into account the direction and magnitude of the internal magnetization due to the evolution of $\xi$. Note that the single scalar variable $\xi$ relates the 6 independent components of the inelastic reorientation tensor $\mathbf{E}^{r}$ and 3 independent components of the magnetization vector $\mathbf{M}^{r}$ through the evolution equations and so the model is capable of taking into account any arbitrary magneto-mechanical loading conditions.

The evolution of interaction or mixing energy between the martensitic variants during reorientation $\left(\dot{g}^{r}\right)$ can be represented by

$\dot{g}^{r}=f^{r} \dot{\xi}$,

where $f^{r}$ is the hardening function.

The total thermodynamic driving force $\pi^{r}$ due to variant reorientation is given by

$$
\pi^{r}=\boldsymbol{\pi}_{\mathbf{E}^{r}}: \boldsymbol{\Lambda}^{r}+\boldsymbol{\pi}_{\mathbf{M}^{r}} \cdot \boldsymbol{\gamma}^{r}+\pi_{\xi}+\pi_{g^{r}} f^{r}
$$

The following transformation function, $\Phi$, is then introduced,

$$
\Phi:=\left\{\begin{array}{cc}
\pi^{r}-Y^{r}, & \dot{\xi}>0 \\
-\pi^{r}-Y^{r}, & \dot{\xi}<0
\end{array}, \quad \Phi \leq 0,\right.
$$

where $Y^{r}$ is a positive scalar associated with the internal dissipation during reorientation. The proposed reorientation function is similar to the transformation function used with conventional shape memory behavior (Lagoudas et al., 1996; Qidwai and Lagoudas, 2000). It is assumed that the constraints of the reorientation process follow the principle of maximum dissipation and can be expressed in terms of the Kuhn Tucker type conditions (Simo and Hughes, 1998)

$$
\Phi \leq 0, \quad \Phi \dot{\xi}=0
$$

\section{Proposed Gibbs free energy and constitutive equations for variant reorientation}

We denote the Gibbs free energy of the variant-1 and the variant-2 by $G^{V_{1}}$ and $G^{V_{2}}$ respectively. The Gibbs free energy of the transforming phase is denoted by $G^{V_{1} \rightarrow V_{2}}$. We write

$$
\begin{aligned}
G\left(\mathbf{S}^{E}, \mathbf{H}, T, \mathbf{E}^{r}, \mathbf{M}^{r}, \xi, g\right)= & G^{V_{1}}\left(\mathbf{S}^{E}, \mathbf{H}, T\right) \\
& +G^{V_{1} \rightarrow V_{2}}\left(\mathbf{S}^{E}, \mathbf{H}, T, \mathbf{E}^{r}, \mathbf{M}^{r}, \xi, g\right)
\end{aligned}
$$

where

$$
\begin{aligned}
G^{V_{1} \rightarrow V_{2}}\left(\mathbf{S}^{E}, \mathbf{H}, T, \mathbf{E}^{r}, \mathbf{M}^{r}, \xi, g\right)= & \xi\left[G^{V_{2}}\left(\mathbf{S}^{E}, \mathbf{H}, T\right)-G^{V_{1}}\left(\mathbf{S}^{E}, \mathbf{H}, T\right)\right] \\
& +G^{I}\left(\mathbf{S}^{E}, \mathbf{H}, \mathbf{E}^{r}, \mathbf{M}^{r}\right)+G^{m i x}(g) .
\end{aligned}
$$

$G^{I}$ and $G^{m i x}$ are the Gibbs free energies due to the magnetoinelastic deformation and the energy due to the mixing of the two variants during reorientation. We determine the integrity basis of the scalar function $G$ for the above mentioned tensorial arguments in the following subsections. Detailed group-theoretical development to obtain the integrity basis for such a single crystal material system can be found in Haldar and Lagoudas (2014). Here we briefly present the applied part of the theoretical development in a aim to calibrate the new model for the stability analysis.

\subsection{Finite symmetry restriction for single crystal}

The most widely used material for variant reorientation mechanism is $\mathrm{Ni}_{2} \mathrm{MnGa}$. The martensitic phase has $10 \mathrm{M}$ structure with $14 / \mathrm{mmm}$ space group. The classical point group is $4 / \mathrm{mmm}\left(D_{4 h}\right)$. The five magnetic point groups are $\underline{4} / \underline{\mathrm{mmm}}, 4 / \underline{\mathrm{mmm}}, \underline{4} / \mathrm{mm} \underline{\mathrm{m}}, 4 / \underline{\mathrm{mmm}}, 4 / \mathrm{mmm}$. Among them only the $4 / \mathrm{mmm}$ is ferromagnetic and the rest of the members are antiferromagnetic (Cracknell, 1975). So we consider $4 / \mathrm{mmm}$ to generate the integrity basis.

There are three variants for tetragonal martensitic phase. We denote variant-3, which has shorter length (c) along the $z$ direction. The $x$ and $y$ axes are along the longer side $a$ (Fig. 1(a)). The stereographic representation of the group elements of $4 / \mathrm{mmm}$ is shown in Fig. 1(b). The notation of the diagram is followed from Bradley and Cracknell (1972). The filled square ( $\boldsymbol{\square}$ ) at the center represents the 4 -fold rotations along the $z$ axis, which are perpendicular to the plane of the paper and obey the right-hand rule. A solid ellipse (๑) denotes 2 -fold rotation along the $i-i$ axis. The alpha-numeric labeling of the symmetry operations are placed on the figure in the position to which the letter $E$ is taken by that operation. $C_{4 z}^{ \pm}$represents $360^{\circ} / 4$ anticlockwise/clockwise rotation along the $z$ axis and $C_{2 i}$ is the $180^{\circ}$ rotation along the $i-i$ axis. We select variant- 1 (shorter axis is along the $X_{1}$ direction) by applying traction on the single crystal along $X_{1}$. The orientation of the initial configuration of variant- 1 is presented in Fig. 2 . The variant- 2 has the shorter length along the $X_{2}$ direction. When the magnetic field intensity is high enough along the direction of spontaneous magnetization ( $X_{2}$ direction), variant- 2 becomes preferred. We assume that these two structural phases are magneto-elastic.

\subsection{Energetics of variant 1}

In this subsection, we discuss the coupled magneto-mechanical energetics of variant-1. The Gibbs free energy is considered as

$$
G^{V_{1}}=G^{V_{1}}\left(\mathbf{S}^{E}, \mathbf{H}, T_{0}\right)
$$

and the elements of the integrity basis are

$$
\begin{aligned}
& I_{1}=H_{1}, I_{2}=H_{2}^{2}+H_{3}^{2}, I_{3}=S_{22}^{E}+S_{33}^{E}, I_{4}=S_{11}^{E} \\
& I_{5}=\left[S_{31}^{E}\right]^{2}+\left[S_{12}^{E}\right]^{2}, I_{6}=\left[S_{23}^{E}\right]^{2}, I_{7}=S_{22}^{E} S_{33}^{E}, I_{8}=H_{2} S_{12}^{E}+H_{3} S_{31}^{E} .
\end{aligned}
$$




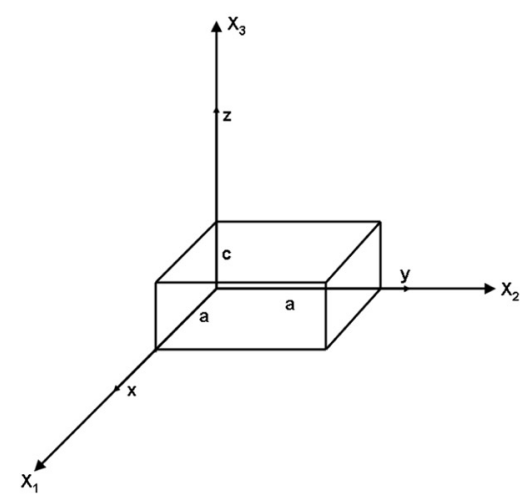

(a)

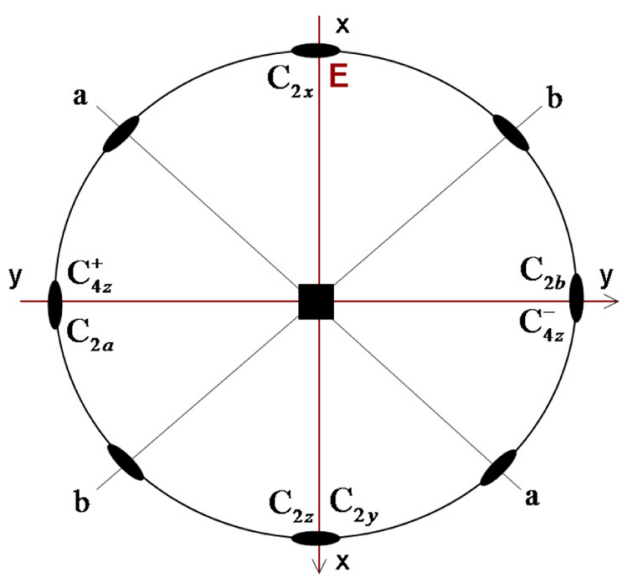

(b)

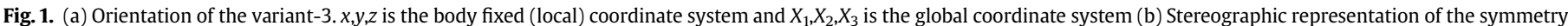
elements and local reference axes for $4 / \mathrm{mmm}$ point group.



Fig. 2. Orientations of variant-1 and variant- 2 .

At a given reference temperature $T_{0}$, we consider the following quadratic expansion

$$
\begin{aligned}
G^{V_{1}}\left(I_{1}, I_{2}, I_{3}, I_{4}, I_{5}, I_{6}, I_{7}, I_{8}\right)= & G_{0}^{1}\left(T_{0}\right)-\frac{1}{\rho_{0}}\left(a_{1} I_{1}+a_{2} I_{1}^{2}+a_{3} I_{2}\right. \\
& +a_{4} I_{3}^{2}+a_{5} I_{4}^{2}+a_{6} I_{5}+a_{7} I_{6}+a_{8} I_{7} \\
& +a_{9} I_{8}+a_{10} I_{1} I_{3}+a_{11} I_{1} I_{4} \\
& \left.+a_{12} I_{3} I_{4}\right)
\end{aligned}
$$

and we write the mechanical and magnetic constitutive equations as ${ }^{1}$

\footnotetext{
${ }^{1}$ The symmetric part of a second order tensor $\mathbf{A}$ is denoted by Sym $[\mathbf{A}]=\frac{1}{2}\left(\mathbf{A}+\mathbf{A}^{T}\right)$.
}

$$
\begin{aligned}
\mathbf{E}^{1}= & -\rho_{0} G^{V_{1}}, \mathbf{S}^{E} \\
= & \left(2 a_{5} I_{4}+a_{12} I_{3}+a_{11} I_{1}\right) \mathbf{i} \otimes \mathbf{i}+2\left(2 a_{6} S_{12}^{E}+a_{9} H_{2}\right) \operatorname{Sym}[\mathbf{i} \otimes \mathbf{j}] \\
& +\left(2 a_{4} I_{3}+a_{8} S_{33}^{E}+a_{12} I_{4}+a_{10} I_{1}\right) \mathbf{j} \otimes \mathbf{j}+4 a_{7} S_{23}^{E} \operatorname{Sym}[\mathbf{j} \otimes \mathbf{k}] \\
& +2\left(2 a_{6} S_{31}^{E}+a_{9} H_{3}\right) \operatorname{Sym}[\mathbf{k} \otimes \mathbf{i}]+\left(a_{8} S_{22}^{E}+a_{12} I_{4}+a_{10} I_{1}\right. \\
& \left.+2 a_{4} I_{3}\right) \mathbf{k} \otimes \mathbf{k}
\end{aligned}
$$

and

$$
\begin{aligned}
\mu_{0} \mathbf{M}^{1}= & -\rho_{0} G^{V_{1}}, \mathbf{H} \\
= & \left(a_{1}+2 a_{2} H_{1}+a_{10}\left(S_{22}^{E}+S_{33}^{E}\right)+a_{11} S_{11}^{E}\right) \mathbf{i}+\left(2 a_{3} H_{2}\right. \\
& \left.+a_{9} S_{12}^{E}\right) \mathbf{j}+\left(2 a_{3} H_{3}+a_{9} S_{31}^{E}\right) \mathbf{k} .
\end{aligned}
$$

\subsection{Energetics of variant-2}

Similarly, considering the Gibbs free energy for variant-2 as

$G^{V_{2}}=G^{V_{2}}\left(\mathbf{S}^{E}, \mathbf{H}, T_{0}\right)$

the elements of the integrity basis are given by

$J_{1}=H_{2}, J_{2}=H_{3}^{2}+H_{1}^{2}, J_{3}=S_{33}^{E}+S_{11}^{E}, J_{4}=S_{22}^{E}$,

$J_{5}=\left[S_{12}^{E}\right]^{2}+\left[S_{23}^{E}\right]^{2}, J_{6}=\left[S_{31}^{E}\right]^{2}, J_{7}=S_{33}^{E} S_{11}^{E}, J_{8}=H_{3} S_{23}^{E}+H_{1} S_{12}^{E}$

It should be noted that the global components of the elements of the integrity basis of variant-2 are different than variant- 1 due to different orientation. $G^{V_{2}}$ can be expanded up to quadratic power as 


$$
\begin{aligned}
G^{V_{2}}\left(J_{1}, J_{2}, J_{3}, J_{4}, J_{5}, J_{6}, J_{7}, J_{8}\right)= & G_{0}^{2}\left(T_{0}\right)-\frac{1}{\rho_{0}}\left(b_{1} J_{1}+b_{2} J_{1}^{2}+b_{3} J_{2}\right. \\
& +b_{4} J_{3}^{2}+b_{5} J_{4}^{2}+b_{6} J_{5}+b_{7} J_{6}+b_{8} J_{7} \\
& +b_{9} J_{8}+b_{10} J_{1} J_{3}+b_{11} J_{1} J_{4} \\
& \left.+b_{12} J_{3} J_{4}\right) .
\end{aligned}
$$

The strain and magnetization can then be expressed as

$$
\begin{aligned}
\mathbf{E}^{2}= & -\rho_{0} G^{V_{2}}, \mathbf{s}^{E} \\
= & \left(2 b_{4} J_{3}+b_{8} S_{33}^{E}+b_{12} J_{4}+b_{10} J_{1}\right) \mathbf{i} \otimes \mathbf{i}+2\left(2 b_{6} S_{12}^{E}+b_{9} H_{1}\right) \\
& \times \operatorname{Sym}[\mathbf{i} \otimes \mathbf{j}]+\left(2 b_{5} J_{4}+b_{12} J_{3}+b_{11} J_{1}\right) \mathbf{j} \otimes \mathbf{j}+2\left(2 b_{6} S_{23}^{E}+b_{9} H_{3}\right) \\
& \times \operatorname{Sym}[\mathbf{j} \otimes \mathbf{k}]+4 b_{7} S_{31}^{E} \operatorname{Sym}[\mathbf{k} \otimes \mathbf{i}] \\
& +\left(2 b_{4} J_{3}+b_{8} S_{11}^{E}+b_{12} J_{4}+b_{10} J_{1}\right) \mathbf{k} \otimes \mathbf{k} .
\end{aligned}
$$

and

$$
\begin{aligned}
\mu_{0} \mathbf{M}^{2}= & -\rho_{0} G^{V_{2}}, \mathbf{H} \\
= & \left(2 b_{3} H_{1}+b_{9} S_{12}^{E}\right) \mathbf{i}+\left(b_{1}+2 b_{2} H_{2}+b_{10}\left(S_{33}^{E}+S_{11}^{E}\right)\right. \\
& \left.+b_{11} S_{22}^{E}\right) \mathbf{j}+\left(2 b_{3} H_{3}+b_{9} S_{23}^{E}\right) \mathbf{k} .
\end{aligned}
$$

\subsection{Reorienting from variant-1 to variant 2}

Considering the initial phase of the single crystal MSMA as the stress favored variant-1 and applying magnetic field, field favored variant-2 nucleates. During the nucleation process, the two variants coexist and form twin structure. The internal strain and magnetization thus generated are taken into account by considering the internal variables $\mathbf{E}^{r}$ and $\mathbf{M}^{r}$. We consider the Gibbs free energy of this intermediate phase as

$G^{I}=G^{I}\left(\mathbf{S}^{E}, \mathbf{H}, \mathbf{E}^{r}, \mathbf{M}^{r}, T_{0}\right)$

Since variant- 2 is nucleating, we impose symmetry restrictions of variant- 2 on the scalar function $G^{I}$. Considering only first order stress and inelastic strain coupling, the elements of the integrity basis can be written as

$K_{1}=S_{33}^{E}+S_{11}^{E}, \quad K_{2}=S_{22}^{E}, \quad K_{3}=E_{33}^{r}+E_{11}^{r}, \quad K_{4}=E_{22}^{r}$

$K_{5}=H_{2}, \quad K_{6}=M_{2}^{r}, \quad K_{7}=\left[S_{12}^{E}\right]^{2}+\left[S_{23}^{E}\right]^{2}, \quad K_{8}=\left[S_{31}^{E}\right]^{2}$

$K_{9}=S_{33}^{E} S_{11}^{E}, \quad K_{10}=\left[E_{12}^{r}\right]^{2}+\left[E_{23}^{r}\right]^{2}, \quad K_{11}=\left[E_{31}^{r}\right]^{2}$

$K_{12}=E_{33}^{r} S_{11}^{E}, \quad K_{13}=S_{31}^{E} E_{31}^{r}, \quad K_{14}=\left[S_{33}^{E}-S_{11}^{E}\right]\left[E_{33}^{r}-E_{11}^{r}\right]$

$K_{15}=E_{12}^{r} S_{12}^{E}+E_{23}^{r} S_{23}^{E}, \quad K_{16}=H_{3}^{2}+H_{1}^{2}, \quad K_{17}=\left[M_{3}^{r}\right]^{2}+\left[M_{1}^{r}\right]^{2}$,

$K_{18}=H_{3} M_{3}^{r}+H_{1} M_{1}^{r}$.

Moreover, considering first order coupling between stress and inelastic strain and between field and internal magnetization, the expanded form of the Gibbs free energy is written as

$$
\begin{aligned}
G^{I} & \left(K_{1}, K_{2}, K_{3}, K_{4}, K_{5}, K_{6}, K_{12}, K_{13}, K_{14}, K_{15}, K_{18}\right) \\
= & G_{0}^{I}\left(T_{0}\right)-\frac{1}{\rho_{0}}\left(c_{1} K_{1} K_{3}+c_{2} K_{1} K_{4}+c_{3} K_{2} K_{3}+c_{4} K_{2} K_{4}+c_{5} K_{5} K_{6}\right. \\
& \left.+c_{6} K_{12}+c_{7} K_{13}+c_{8} K_{14}+c_{9} K_{15}+c_{10} K_{18}\right) .
\end{aligned}
$$

The constitutive equations for the above proposed scalar function $G^{I}$ are given by

$$
\begin{aligned}
\mathbf{E}^{I}= & -\rho_{0} G^{I}, \mathbf{s}^{E} \\
= & {\left[c_{1}\left(E_{33}^{r}+E_{11}^{r}\right)+c_{2} E_{22}^{r}-c_{8}\left(E_{33}^{r}-E_{11}^{r}\right)+c_{6} E_{33}^{r}\right] \mathbf{i} \otimes \mathbf{i} } \\
& +2 c_{9} E_{12}^{r} \operatorname{Sym}[\mathbf{i} \otimes \mathbf{j}]+\left[c_{3}\left(E_{33}^{r}+E_{11}^{r}\right)+c_{4} E_{22}^{r}\right] \mathbf{j} \otimes \mathbf{j} \\
& +2 c_{9} E_{23}^{r} \operatorname{Sym}[\mathbf{j} \otimes \mathbf{k}]+2 c_{7} E_{31}^{r} \operatorname{Sym}[\mathbf{k} \otimes \mathbf{i}]+\left[c_{1}\left(E_{33}^{r}+E_{11}^{r}\right)\right. \\
& \left.+c_{2} E_{22}^{r}+c_{8}\left(E_{33}^{r}-E_{11}^{r}\right)\right] \mathbf{k} \otimes \mathbf{k},
\end{aligned}
$$

and

$$
\begin{aligned}
\mu_{0} \mathbf{M}^{I} & =-\rho_{0} G^{I}, \mathbf{H}=c_{5} K_{6} K_{5, \mathbf{H}}+c_{10} K_{18, \mathbf{H}} \\
& =c_{5} M_{2}^{r} \mathbf{j}+c_{10}\left(M_{1}^{r} \mathbf{i}+M_{3}^{r} \mathbf{k}\right) .
\end{aligned}
$$

\subsubsection{Evolution equations}

As the strain and magnetization evolution equations are concerned, we write the strain evolution equation as

$\dot{E}_{i j}^{r}=\Lambda_{i j}^{r}\left(\mathbf{S}^{\prime E}\right) \dot{\xi}$

It can be shown that $\Lambda_{i j}^{r}$ can be spanned as (Haldar and Lagoudas, 2014)

$\Lambda_{i j}^{r}=\sum_{p=1}^{m} d_{p}(\{I\}) \mathscr{P}_{i j}^{p}$,

where, $d_{p}$ 's are the scalar polynomials, $\{I\}=\left\{S_{33}^{\prime E}+S_{11}^{\prime E}, S_{22}^{\prime E},\left(S_{31}^{\prime E}\right)^{2},\left(S_{33}^{\prime E}-S_{11}^{\prime E}\right)^{2},\left(S_{12}^{\prime E}\right)^{2}+\left(S_{23}^{\prime E}\right)^{2}\right\}$ and the elements of the set $\{\mathscr{D}\}$ are given by

$\mathscr{D}^{1}=\mathbf{i} \otimes \mathbf{i}+\mathbf{k} \otimes \mathbf{k}, \quad \mathscr{D}^{2}=\mathbf{j} \otimes \mathbf{j}, \quad \mathscr{D}^{3}=2 S_{31}^{\prime E} \operatorname{Sym}[\mathbf{i} \otimes \mathbf{k}]$, $\mathscr{D}^{4}=\left(S_{33}^{E}-S_{11}^{\prime E}\right)(\mathbf{k} \otimes \mathbf{k}-\mathbf{i} \otimes \mathbf{i})$, $\mathscr{D}^{5}=2 S_{12}^{\prime E} \operatorname{Sym}[\mathbf{i} \otimes \mathbf{j}]+2 S_{23}^{\prime E} \operatorname{Sym}[\mathbf{j} \otimes \mathbf{k}]$.

Thus, spanning $\Lambda^{r}$ up to $m=5$ in terms of the elements of $\{\mathscr{D}\}$, we write

$$
\begin{aligned}
\Lambda^{r}= & d_{1}(\mathbf{i} \otimes \mathbf{i}+\mathbf{k} \otimes \mathbf{k})+d_{2} \mathbf{j} \otimes \mathbf{j}+2 d_{3} S_{31}^{E} \operatorname{Sym}[\mathbf{i} \otimes \mathbf{k}] \\
& +d_{4}\left(S_{33}^{\prime E}-S_{11}^{E}\right)(\mathbf{k} \otimes \mathbf{k}-\mathbf{i} \otimes \mathbf{i})+2 d_{5}\left(S_{12}^{\prime E} \operatorname{Sym}[\mathbf{i} \otimes \mathbf{j}]\right. \\
& \left.+S_{23}^{\prime E} \operatorname{Sym}[\mathbf{j} \otimes \mathbf{k}]\right) .
\end{aligned}
$$

Similarly, for the magnetization evolution equation

$\dot{\mathbf{M}}^{r}=\boldsymbol{\gamma}_{4}^{r}\left(\mathbf{S}^{\mathrm{E}}\right) \dot{\xi}$,

we can write

$\gamma_{i}^{r}=\sum_{p=1}^{m} d_{P}^{\prime}(\{I\}) \mathscr{D}_{i}^{p}$,

where $\{I\}=\left\{S_{33}^{\prime E}+S_{11}^{\prime E},\left(S_{12}^{\prime E}\right)^{2}+\left(S_{23}^{\prime E}\right)^{2}\right\}$ and 
$\mathscr{D}^{1}=\mathbf{j}, \quad \mathscr{D}^{2}=S_{12}^{\prime E} \mathbf{i}+S_{23}^{\prime E} \mathbf{k}$.

The expression for $\gamma^{r}$ is then given by

$$
\boldsymbol{\gamma}^{r}=d_{1}^{\prime} \mathbf{j}+d_{2}^{\prime}\left(S_{12}^{E} \mathbf{i}+S_{23}^{E} \mathbf{k}\right)
$$

\subsubsection{Mixing energy}

We consider the Gibbs free energy for the mixing as

$G^{m i x}\left(g^{r}\right)=-\frac{1}{\rho} g^{r}$.

The evolution of the reorientation hardening energy $g^{r}$ is related with a hardening function $f^{r}$. The selection of such a function is discussed in B.1.3 for a specific example.

\subsection{Vectorization of matrices}

The final combined forms of the strain and magnetization constitutive equations are written as

$\mathbf{E}=-\rho_{0} G_{\mathbf{S}^{E}}=\mathbf{E}^{1}+\xi(\Delta \mathbf{E})+\mathbf{E}^{I}$,

$\mathbf{M}=-\frac{\rho_{0}}{\mu_{0}} G_{, \mathbf{H}}=\mathbf{M}^{1}+\xi(\Delta \mathbf{M})+\mathbf{M}^{I}$,

where $\Delta \mathbf{E}=\mathbf{E}^{2}-\mathbf{E}^{1}$ and $\Delta \mathbf{M}=\mathbf{M}^{2}-\mathbf{M}^{1}$.

At this point we consider vectorization of matrices $[\mathbf{E}]$ and $\left[\mathbf{S}^{E}\right]$, which represents the constitutive equations in a convenient form that is suitable for stability analysis. Moreover, since $[\mathbf{E}]$ and $\left[\mathbf{S}^{E}\right]$ are symmetric matrices, we consider half-vectorization. In a general way, half-vectorization of a $n \times n$ symmetric matrix $\mathbb{A}$ is denoted by

$\operatorname{vech}(\mathbb{A})=\left[\mathbb{A}_{11}, \ldots, \mathbb{A}_{n 1}, \mathbb{A}_{22}, \ldots, \mathbb{A}_{n 2}, \ldots, \mathbb{A}_{(n-1)(n-1)}, \mathbb{A}_{(n-1) n}, \mathbb{A}_{n n}\right]^{T}$

We write for simplicity $\operatorname{vech}(\mathbb{A})=(\mathbb{A})$ and so

$\left(\mathbf{E}^{1}\right)=\left[\mathbf{A}_{s}\right]\left(\mathbf{S}^{E}\right)+\left[\mathbf{A}_{h}\right](\mathbf{H})$,

$\left(\mathbf{E}^{2}\right)=\left[\mathbf{B}_{s}\right]\left(\mathbf{S}^{E}\right)+\left[\mathbf{B}_{h}\right](\mathbf{H})$.

Here $\left[\mathbf{A}_{s}\right]_{6 \times 6},\left[\mathbf{B}_{s}\right]_{6 \times 6}$ and $\left[\mathbf{A}_{h}\right]_{6 \times 3},\left[\mathbf{B}_{h}\right]_{6 \times 3}$ can be determined from the constitutive responses (10) and (13). $(\cdot)$ is $6 \times 1$ for a symmetric tensor and $3 \times 1$ for a vector. Thus we write (19) as

$$
\begin{aligned}
(\mathbf{E}) & =\left(\mathbf{E}^{1}\right)+\xi\left(\left(\mathbf{E}^{2}\right)-\left(\mathbf{E}^{1}\right)\right)+\left(\mathbf{E}^{I}\right) \\
& =\left[\mathbf{A}_{s}\right]\left(\mathbf{S}^{E}\right)+\xi\left[\mathbf{T}_{1}\right]\left(\mathbf{S}^{E}\right)+\left[\mathbf{A}_{h}\right](\mathbf{H})+\xi\left[\mathbf{T}_{2}\right](\mathbf{H})+\left(\mathbf{E}^{I}\right),
\end{aligned}
$$

where

$\left[\mathbf{T}_{1}\right]=\left[\mathbf{B}_{s}\right]-\left[\mathbf{A}_{s}\right], \quad\left[\mathbf{T}_{2}\right]=\left[\mathbf{B}_{h}\right]-\left[\mathbf{A}_{h}\right]$.

Similarly, we can write from (11) and (14)

$\left(\mathbf{M}^{1}\right)=\left[\mathbf{A}_{m s}\right]\left(\mathbf{S}^{E}\right)+\left[\mathbf{A}_{m h}\right](\mathbf{H})+(\mathbf{p})$

$\left(\mathbf{M}^{2}\right)=\left[\mathbf{B}_{m s}\right]\left(\mathbf{S}^{E}\right)+\left[\mathbf{B}_{m h}\right](\mathbf{H})+(\mathbf{q})$,

where, the dimensions of the above matrices are $\left[\mathbf{A}_{m s}\right]_{3} \times 6$, $\left[\mathbf{B}_{m s}\right]_{3 \times 6},\left[\mathbf{A}_{m h}\right]_{3 \times 3}$ and $\left[\mathbf{B}_{m h}\right]_{3} \times 3$ and from (20)

$$
\begin{aligned}
(\mathbf{M})= & \left(\mathbf{M}^{1}\right)+\xi\left(\left(\mathbf{M}^{2}\right)-\left(\mathbf{M}^{1}\right)\right)+\left(\mathbf{M}^{I}\right) \\
= & {\left[\mathbf{A}_{m s}\right]\left(\mathbf{S}^{E}\right)+\xi\left[\mathbf{T}_{1 m}\right]\left(\mathbf{S}^{E}\right)+\left[\mathbf{A}_{m h}\right](\mathbf{H})+\xi\left[\mathbf{T}_{2 m}\right](\mathbf{H})+\left(\mathbf{T}_{3 m}\right) \xi } \\
& +\left(\mathbf{M}^{I}\right)
\end{aligned}
$$

where

$$
\begin{aligned}
{\left[\mathbf{T}_{1 m}\right] } & =\left[\mathbf{B}_{m s}\right]-\left[\mathbf{A}_{m s}\right], \quad\left[\mathbf{T}_{2 m}\right]=\left[\mathbf{B}_{m h}\right]-\left[\mathbf{A}_{m h}\right], \quad\left(\mathbf{T}_{3 m}\right) \\
& =(\mathbf{q})-(\mathbf{p}) .
\end{aligned}
$$

\section{Tangent stiffness and incremental magneto-mechanical constitutive equations}

We now derive the tangent stiffness for the incremental magneto-mechanical constitutive equations. By taking the time derivative of Eq. (23) and write

$$
\begin{aligned}
(\dot{\mathbf{E}})= & {\left[\mathbf{A}_{s}\right]\left(\dot{\mathbf{S}}^{E}\right)+\dot{\xi}\left[\mathbf{T}_{1}\right]\left(\mathbf{S}^{E}\right)+\xi\left[\mathbf{T}_{1}\right]\left(\dot{\mathbf{S}}^{E}\right)+\left[\mathbf{A}_{h}\right](\dot{\mathbf{H}})+\dot{\xi}\left[\mathbf{T}_{2}\right](\mathbf{H}) } \\
& +\xi\left[\mathbf{T}_{2}\right](\dot{\mathbf{H}})+\dot{\mathbf{E}}^{I} \\
= & {\left[\left[\mathbf{A}_{S}\right]+\xi\left[\mathbf{T}_{2}\right]\right]\left(\dot{\mathbf{S}}^{E}\right)+\left[\left[\mathbf{A}_{h}\right]+\xi\left[\mathbf{T}_{2}\right]\right](\dot{\mathbf{H}})+\left(\left[\mathbf{T}_{1}\right]\left(\mathbf{S}^{E}\right)\right.} \\
& \left.+\left[\mathbf{T}_{2}\right](\mathbf{H})+(\boldsymbol{\Lambda})\right) \dot{\xi}
\end{aligned}
$$

or

$$
\left(\dot{\mathbf{S}}^{E}\right)=[\mathbb{C}](\dot{\mathbf{E}})+[\mathbb{P}](\dot{\mathbf{H}})+(\mathbb{D}) \dot{\xi},
$$

where $[\mathbb{C}]=\left[\left[\mathbf{A}_{s}\right]+\xi\left[\mathbf{T}_{1}\right]\right]^{-1},[\mathbb{P}]=-\left[\left[\mathbf{A}_{s}\right]+\xi\left[\mathbf{T}_{1}\right]\right]^{-1}\left[\left[\mathbf{A}_{h}\right]+\xi\left[\mathbf{T}_{2}\right]\right]$ and $(\mathbb{D})=-\left[\left[\mathbf{A}_{s}\right]+\xi\left[\mathbf{T}_{1}\right]\right]^{-1}\left(\left[\mathbf{T}_{1}\right]\left(\mathbf{S}^{E}\right)+\left[\mathbf{T}_{2}\right](\mathbf{H})+(\boldsymbol{\Lambda})\right)$.

Similarly,

$$
\begin{aligned}
(\dot{\mathbf{M}})= & {\left[\mathbf{A}_{m s}\right]\left(\dot{\mathbf{S}}^{E}\right)+\dot{\xi}\left[\mathbf{T}_{1 m}\right]\left(\mathbf{S}^{E}\right)+\xi\left[\mathbf{T}_{1 m}\right]\left(\dot{\mathbf{S}}^{E}\right)+\left[\mathbf{A}_{m h}\right](\dot{\mathbf{H}}) } \\
& +\dot{\xi}\left[\mathbf{T}_{2 m}\right](\mathbf{H})+\xi\left[\mathbf{T}_{2 m}\right](\dot{\mathbf{H}})+\dot{\xi}\left(\mathbf{T}_{3 m}\right)+\dot{\mathbf{M}}^{I} \\
= & {\left[\left[\mathbf{A}_{m s}\right]+\xi\left[\mathbf{T}_{1 m}\right]\right]\left(\dot{\mathbf{S}}^{E}\right)+\left[\left[\mathbf{A}_{m h}\right]+\xi\left[\mathbf{T}_{2 m}\right]\right](\dot{\mathbf{H}})+\left(\left[\mathbf{T}_{1 m}\right]\left(\mathbf{S}^{E}\right)\right.} \\
& \left.+\left[\mathbf{T}_{2 m}\right](\mathbf{H})+\left(\mathbf{T}_{3 m}\right)+(\boldsymbol{\gamma})\right) \dot{\xi}
\end{aligned}
$$

or

$$
(\dot{\mathbf{M}})=\left[\mathbb{C}_{m}\right]\left(\dot{\boldsymbol{S}}^{E}\right)+\left[\mathbb{P}_{m}\right](\dot{\mathbf{H}})+\left(\mathbb{D}_{m}\right) \dot{\xi}
$$

where $\quad\left[\mathbb{C}_{m}\right]=\left[\mathbf{A}_{m s}\right]+\xi\left[\mathbf{T}_{1 m}\right], \quad\left[\mathbb{P}_{m}\right]=\left[\mathbf{A}_{m h}\right]+\xi\left[\mathbf{T}_{2 m}\right] \quad$ and $\left(\mathbb{D}_{m}\right)=\left(\left[\mathbf{T}_{1 m}\right]\left(\mathbf{S}^{E}\right)+\left[\mathbf{T}_{2 m}\right](\mathbf{H})+\left(\mathbf{T}_{3 m}\right)+(\boldsymbol{\gamma})\right)$. Substituting the relation (26) in (27) we obtain

$$
\begin{aligned}
(\dot{\mathbf{M}}) & =\left[\mathbb{C}_{m}\right]\left(\dot{\mathbf{S}}^{E}\right)+\left[\mathbb{P}_{m}\right](\dot{\mathbf{H}})+\left(\mathbb{D}_{m}\right) \dot{\xi} \\
& =\left[\mathbb{C}_{m}\right]([\mathbb{C}](\dot{\mathbf{E}})+[\mathbb{P}](\dot{\mathbf{H}})+(\mathbb{D}) \dot{\xi})+\left[\mathbb{P}_{m}\right](\dot{\mathbf{H}})+\left(\mathbb{D}_{m}\right) \dot{\xi} \\
& =\left[\mathbb{C}_{m}\right][\mathbb{C}](\dot{\mathbf{E}})+\left(\left[\mathbb{C}_{m}\right][\mathbb{P}]+\left[\mathbb{P}_{m}\right]\right)(\dot{\mathbf{H}})+\left(\left[\mathbb{C}_{m}\right](\mathbb{D})+\left(\mathbb{D}_{m}\right)\right) \dot{\xi} \\
& =\left[\mathbb{C}_{m}^{\prime}\right](\dot{\mathbf{E}})+\left[\mathbb{P}_{m}^{\prime}\right](\dot{\mathbf{H}})+\left(\mathbb{D}_{m}^{\prime}\right) \dot{\xi}
\end{aligned}
$$

Now, from the consistency condition (8) we consider two cases. Case-I: $\dot{\xi} \neq 0$ and $\Phi=0$. 
We write

$$
\begin{gathered}
\dot{\Phi}\left(\mathbf{S}^{E}, \mathbf{H}, \xi\right)=0 \\
\Rightarrow\left(\Phi, \mathbf{S}^{E}\right) \cdot\left(\dot{\mathbf{S}}^{E}\right)+\Phi, \mathbf{H} \cdot \dot{\mathbf{H}}+\Phi, \xi \dot{\xi}=0
\end{gathered}
$$

and replacing $\dot{\mathbf{S}}^{E}$ from (26) we obtain

$$
\begin{gathered}
\left(\Phi, \mathbf{S}^{E}\right) \cdot([\mathbb{C}](\dot{\mathbf{E}})+(\mathbb{D}) \dot{\xi})+\left([\mathbb{P}]^{T} \boldsymbol{\Phi}, \mathbf{S}^{E}+\boldsymbol{\Phi}, \mathbf{H}\right) \cdot \dot{\mathbf{H}}+\Phi, \xi \dot{\xi}=0 \\
\Rightarrow \dot{\xi}=-\frac{\left(\boldsymbol{\Phi}, \mathbf{S}^{E}\right) \cdot[\mathbb{C}] \dot{\mathbf{E}}+\left([\mathbb{P}]^{T} \boldsymbol{\Phi}, \mathbf{S}^{E}+\boldsymbol{\Phi}, \mathbf{H}\right) \cdot \dot{\mathbf{H}}}{\left(\boldsymbol{\Phi}, \mathbf{S}^{E}\right) \cdot(\mathbb{D})+\boldsymbol{\Phi}, \xi}
\end{gathered}
$$

Substituting back (30) in (26) we have

$$
\begin{aligned}
\left(\dot{\mathbf{S}}^{E}\right)= & {[\mathbb{C}](\dot{\mathbf{E}})+[\mathbb{P}](\dot{\mathbf{H}}) } \\
& -(\mathbb{D}) \frac{\left(\Phi, \mathbf{S}^{E}\right) \cdot[\mathbb{C}](\dot{\mathbf{E}})+\left([\mathbb{P}]^{T} \boldsymbol{\Phi}, \mathbf{S}^{E}+\boldsymbol{\Phi}, \mathbf{H}\right) \cdot \dot{\mathbf{H}}}{\left(\boldsymbol{\Phi}, \mathbf{S}^{E}\right) \cdot(\mathbb{D})+\boldsymbol{\Phi}, \xi} \\
= & {\left[[\mathbb{C}]-\frac{(\mathbb{D}) \otimes[\mathbb{C}]^{T} \boldsymbol{\Phi}, \mathbf{S}^{E}}{\left(\boldsymbol{\Phi}, \mathbf{S}^{E}\right) \cdot(\mathbb{D})+\boldsymbol{\Phi}, \xi}\right] \cdot(\dot{\mathbf{E}}) } \\
& +\left[[\mathbb{P}]-\frac{(\mathbb{D}) \otimes\left([\mathbb{P}]^{T} \boldsymbol{\Phi}, \mathbf{S}^{E}+\boldsymbol{\Phi}, \mathbf{H}\right)}{\left(\boldsymbol{\Phi}, \mathbf{S}^{E}\right) \cdot(\mathbb{D})+\boldsymbol{\Phi}, \xi}\right] \cdot \dot{\mathbf{H}} \\
= & {[\mathbb{L}] \cdot(\dot{\mathbf{E}})+[\mathbb{K}] \cdot \dot{\mathbf{H}} }
\end{aligned}
$$

Here $[\mathbb{L}]$ is a $6 \times 6$ mechanical tangent stiffness matrix and $[\mathbb{K}]$ is a $6 \times 3$ magnetic stiffness matrix. Moreover, substituting back the expression of $\dot{\xi}$ in (28) we get

$$
\begin{aligned}
(\dot{\mathbf{M}})= & {\left[\mathbb{C}_{m}^{\prime}\right](\dot{\mathbf{E}})+\left[\mathbb{P}_{m}^{\prime}\right](\dot{\mathbf{H}}) } \\
& -\left(\mathbb{D}_{m}^{\prime}\right) \frac{\left(\boldsymbol{\Phi}, \mathbf{S}^{E}\right) \cdot[\mathbb{C}] \dot{\mathbf{E}}+\left([\mathbb{P}]^{T} \boldsymbol{\Phi}, \mathbf{s}^{E}+\boldsymbol{\Phi}, \mathbf{H}\right) \cdot \dot{\mathbf{H}}}{\left(\boldsymbol{\Phi}, \mathbf{S}^{E}\right) \cdot(\mathbb{D})+\boldsymbol{\Phi}, \xi} \\
= & {\left[\left[\mathbb{C}_{m}^{\prime}\right]-\frac{\left(\mathbb{D}_{m}^{\prime}\right) \otimes[\mathbb{C}]^{T} \boldsymbol{\Phi}, \mathbf{S}^{E}}{\left(\boldsymbol{\Phi}, \mathbf{S}^{E}\right) \cdot(\mathbb{D})+\boldsymbol{\Phi}, \xi} \cdot(\mathbf{E})\right.} \\
& +\left[\left[\mathbb{P}_{m}^{\prime}\right]-\frac{\left(\mathbb{D}_{m}^{\prime}\right) \otimes\left([\mathbb{P}]^{T} \boldsymbol{\Phi}, \mathbf{S}^{E}+\boldsymbol{\Phi}, \mathbf{H}\right)}{\left(\boldsymbol{\Phi}, \mathbf{S}^{E}\right) \cdot(\mathbb{D})+\boldsymbol{\Phi}, \xi}\right] \cdot \dot{\mathbf{H}} \\
= & {\left[\mathbb{L}^{\prime}\right] \cdot(\dot{\mathbf{E}})+\left[\mathbb{K}^{\prime}\right] \cdot \dot{\mathbf{H}} }
\end{aligned}
$$

where $\left[\mathbb{L}^{\prime}\right]$ is a $3 \times 6$ mechanical tangent stiffness matrix and $\left[\mathbb{K}^{\prime}\right]$ is a $3 \times 3$ magnetic stiffness matrix.

Case-II: $\dot{\xi}=0$ and $\Phi<0$

In this case, $[\mathbb{L}]=[\mathbb{C}],[\mathbb{K}]=[\mathbb{P}],\left[\mathbb{L}^{\prime}\right]=\left[\mathbb{C}_{m}^{\prime}\right]$ and $\left[\mathbb{K}^{\prime}\right]=\left[\mathbb{P}_{m}^{\prime}\right]$.

\subsection{Small strain approximation and 2-D reduction of the problem}

Since, the experiments are performed in small strains, we reduce the model into an infinitesimal strain model such that $\mathbf{E} \approx \boldsymbol{\varepsilon}$, $\mathbf{S}^{E} \approx \boldsymbol{\sigma}^{E}=\boldsymbol{\sigma}+\mu_{0} \mathbf{H} \otimes \mathbf{M}, \mathbf{H} \approx \mathbf{h}$ and $\mathbf{M} \approx \mathbf{m}$. We further reduce the problem in 2-D, where the stress components are $\sigma_{11}^{E}, \sigma_{22}^{E}, \sigma_{12}^{E}$, strain components are $\varepsilon_{11}, \varepsilon_{22}, \varepsilon_{12}$, the magnetic field components are $H_{1}, H_{2}$, and the magnetizations are $M_{1}, M_{2}$. The reduced form of the strain constitutive equations in the matrix and vector notation thus can be written as below

$$
\left(\begin{array}{c}
\varepsilon_{11}^{1} \\
\varepsilon_{12}^{1} \\
\varepsilon_{22}^{1}
\end{array}\right)=\left[\begin{array}{ccc}
2 a_{5} & 0 & a_{12} \\
0 & 0 & 2 a_{6} \\
a_{12} & 0 & 2 a_{4}
\end{array}\right]\left(\begin{array}{l}
\sigma_{11}^{E} \\
\sigma_{12}^{E} \\
\sigma_{22}^{E}
\end{array}\right)+\left[\begin{array}{cc}
a_{11} & 0 \\
0 & a_{9} \\
a_{10} & 0
\end{array}\right]\left(\begin{array}{l}
H_{1} \\
H_{2}
\end{array}\right),
$$

$\left(\begin{array}{l}\varepsilon_{11}^{2} \\ \varepsilon_{12}^{2} \\ \varepsilon_{22}^{2}\end{array}\right)=\left[\begin{array}{ccc}2 b_{4} & 0 & b_{12} \\ 0 & 0 & 2 b_{6} \\ b_{12} & 0 & 2 b_{5}\end{array}\right]\left(\begin{array}{l}\sigma_{11}^{E} \\ \sigma_{12}^{E} \\ \sigma_{22}^{E}\end{array}\right)+\left[\begin{array}{cc}0 & b_{10} \\ b_{9} & 0 \\ 0 & b_{11}\end{array}\right]\left(\begin{array}{l}H_{1} \\ H_{2}\end{array}\right)$.

The expressions of $\left[\mathbf{A}_{s}\right],\left[\mathbf{A}_{h}\right]$ and $\left[\mathbf{B}_{s}\right],\left[\mathbf{B}_{h}\right]$, as discussed in Eqs. (21) and (22) respectively, can be found directly from the above two relations for the 2-D case. Moreover, with the above mentioned assumptions, Eq. (16) can be reduced to

$$
\boldsymbol{\varepsilon}^{I}=\left[c_{1} \varepsilon_{11}^{r}+c_{2} \varepsilon_{22}^{r}+c_{8} \varepsilon_{11}^{r}\right] \mathbf{i} \otimes \mathbf{i}+\left[c_{3} \varepsilon_{11}^{r}+c_{4} \varepsilon_{22}^{r}\right] \mathbf{j} \otimes \mathbf{j} .
$$

Enforcing the isochoric condition, $\operatorname{tr}\left(\varepsilon^{I}\right)=0$, we can write $\varepsilon_{22}^{r}=$ $-\varepsilon_{11}^{r}\left[\frac{c_{1}+c_{3}+c_{8}}{c_{2}+c_{4}}\right]$ and obtain

$$
\left(\begin{array}{l}
\varepsilon_{11}^{1} \\
\varepsilon_{12}^{1} \\
\varepsilon_{22}^{1}
\end{array}\right)=\varepsilon_{11}^{r} t_{1}\left(\begin{array}{c}
1 \\
0 \\
-1
\end{array}\right)
$$

where $t_{1}=\frac{c_{1} c_{4}+c_{4} c_{8}-c_{2} c_{3}}{c_{2}+c_{4}}$. Similarly, the matrix forms of the magnetization equations are

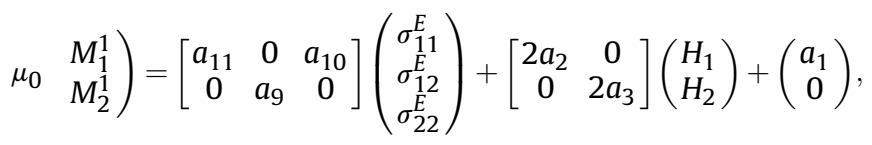

$$
\left.\mu_{0} \begin{array}{c}
M_{1}^{2} \\
M_{2}^{2}
\end{array}\right)=\left[\begin{array}{ccc}
0 & b_{9} & 0 \\
b_{10} & 0 & b_{11}
\end{array}\right]\left(\begin{array}{c}
\sigma_{11}^{E} \\
\sigma_{12}^{E} \\
\sigma_{22}^{E}
\end{array}\right)+\left[\begin{array}{cc}
2 b_{3} & 0 \\
0 & 2 b_{2}
\end{array}\right]\left(\begin{array}{c}
H_{1} \\
H_{2}
\end{array}\right)+\left(\begin{array}{c}
0 \\
b_{1}
\end{array}\right)
$$

The expressions of $\left[\mathbf{A}_{m s}\right],\left[\mathbf{A}_{m h}\right],(\mathbf{p})$ and $\left[\mathbf{B}_{m s}\right],\left[\mathbf{B}_{m h}\right],(\mathbf{q})$ as mentioned in Eq. (24) and (25) respectively, can be obtained from the above two relations. The components form of the internal magnetization can be expressed as

$$
\left.\begin{array}{l}
M_{1}^{I} \\
M_{2}^{I}
\end{array}\right)=\left(\begin{array}{c}
c_{10} M_{1}^{r} \\
c_{5} M_{2}^{r}
\end{array}\right)=\left(\begin{array}{c}
c_{10} d_{1}^{\prime} \sigma_{11}^{\prime E} \\
c_{5} d_{1}^{\prime}
\end{array}\right)=\left(\begin{array}{c}
q^{\prime} \\
p^{\prime}
\end{array}\right) \text {. }
$$

Finally, the 2-D reduction of (31) is written

$$
\left[\begin{array}{l}
\dot{\sigma}_{11}^{E} \\
\dot{\sigma}_{12}^{E} \\
\dot{\sigma}_{22}^{E}
\end{array}\right]=\left[\begin{array}{lll}
L_{11} & L_{12} & L_{13} \\
L_{21} & L_{22} & L_{23} \\
L_{31} & L_{32} & L_{33}
\end{array}\right]\left[\begin{array}{l}
\dot{\varepsilon}_{11} \\
\dot{\varepsilon}_{12} \\
\dot{\varepsilon}_{22}
\end{array}\right]+\left[\begin{array}{ll}
K_{11} & K_{12} \\
K_{21} & K_{22} \\
K_{31} & K_{32}
\end{array}\right]\left[\begin{array}{l}
\dot{H}_{1} \\
\dot{H}_{2}
\end{array}\right]
$$

and Eq. (32) reads

$$
\left[\begin{array}{l}
\dot{M}_{1} \\
\dot{M}_{2}
\end{array}\right]=\left[\begin{array}{lll}
L_{11}^{\prime} & L_{12}^{\prime} & L_{13}^{\prime} \\
L_{21}^{\prime} & L_{22}^{\prime} & L_{23}^{\prime}
\end{array}\right]\left[\begin{array}{l}
\dot{\varepsilon}_{11} \\
\dot{\varepsilon}_{12} \\
\dot{\varepsilon}_{22}
\end{array}\right]+\left[\begin{array}{ll}
K_{11}^{\prime} & K_{12}^{\prime} \\
K_{21}^{\prime} & K_{22}^{\prime}
\end{array}\right]\left[\begin{array}{l}
\dot{H}_{1} \\
\dot{H}_{2}
\end{array}\right]
$$

It should be noted that if we consider plane stress problem, the out of plane strain needs to be calculated and under plane strain the out of plane stress needs to be calculated and also the material parameters have a different interpretation. 


\section{2-D stability analysis of the magneto-mechanical coupled system}

Before performing stability analysis, we first list the system of equations below

$\nabla \cdot \mathbf{H}+\nabla \cdot \mathbf{M}=0$

$\nabla \times \mathbf{H}=0$

$\nabla \cdot \boldsymbol{\sigma}^{E}+\mu_{0}(\nabla \cdot \mathbf{H}) \mathbf{H}=0$

$\boldsymbol{\sigma}^{E}=[\mathbb{L}](\dot{\boldsymbol{\varepsilon}})+[\mathbb{K}] \dot{\mathbf{H}}$

$\dot{\mathbf{M}}=\left[\mathbb{L}^{\prime}\right](\dot{\boldsymbol{\varepsilon}})+\left[\mathbb{K}^{\prime}\right] \dot{\mathbf{H}}$

$\varepsilon=\frac{1}{2}\left(\Psi+\Psi^{T}\right)$

$\nabla \times \Psi=0$.

We introduce a tensor potential $\Psi=\nabla \otimes \mathbf{u}$, where $\mathbf{u}$ is the displacement. Introducing a potential $\Psi$ allows us to express the coupled system as a system of first order PDEs. It should be noted that we replaced $\mathbf{B}$ from the Gauss Law, i.e, $\nabla \cdot \mathbf{B}=0$, by means of the constitutive equation $\mathbf{B}=\mu_{0}(\mathbf{M}+\mathbf{H})$ and also $\operatorname{skw}\left(\boldsymbol{\sigma}^{E}\right)=0$ since $\boldsymbol{\sigma}^{E}$ is symmetric.

These are 14 equations (in 2-D) and $H_{1}, H_{2}, M_{1}, M_{2}, \sigma_{11}^{E}, \sigma_{12}^{E}, \sigma_{22}^{E}$, $\varepsilon_{11}, \varepsilon_{12}, \varepsilon_{22}, \Psi_{11}, \Psi_{12}, \Psi_{21}, \Psi_{22}$ are the 14 unknowns. We can write from (41f)

$\varepsilon_{11}=\Psi_{11}, \quad \varepsilon_{22}=\Psi_{22}, \quad \varepsilon_{12}=\frac{1}{2}\left(\Psi_{12}+\Psi_{21}\right)$

and so

$[\boldsymbol{\varepsilon}]=\left[\begin{array}{cc}\Psi_{11} & \frac{1}{2}\left(\Psi_{12}+\Psi_{21}\right) \\ \frac{1}{2}\left(\Psi_{21}+\Psi_{12}\right) & \Psi_{22}\end{array}\right]$.

Taking the time derivative we can further write

$\left(\begin{array}{l}\dot{\varepsilon}_{11} \\ \dot{\varepsilon}_{12} \\ \dot{\varepsilon}_{22}\end{array}\right)=\left[\begin{array}{llll}1 & 0 & 0 & 0 \\ 0 & 0 & \frac{1}{2} & \frac{1}{2} \\ 0 & 1 & 0 & 0\end{array}\right]\left(\begin{array}{c}\dot{\Psi}_{11} \\ \dot{\Psi}_{22} \\ \dot{\Psi}_{12} \\ \dot{\Psi}_{21}\end{array}\right)$

or

$(\dot{\boldsymbol{\varepsilon}})=[\mathscr{E}](\dot{\boldsymbol{\Psi}})$.

Thus, (41d) becomes

$\left(\dot{\boldsymbol{\sigma}}^{E}\right)=[\mathbb{L}][\mathscr{E}](\dot{\mathbf{\Psi}})+[\mathbb{K}](\dot{\mathbf{H}})$

and (41e) becomes

$\dot{\mathbf{M}}=\left[\mathbb{L}^{\prime}\right][\mathscr{E}](\dot{\mathbf{\Psi}})+\left[\mathbb{K}^{\prime}\right](\dot{\mathbf{H}})$.

Finally, from (45) with the help of (41c), (46) with the help of (41a), (41b) and (41g), we eliminate $\boldsymbol{\sigma}^{E}$ and $\mathbf{M}$ respectively and write the system of equations as

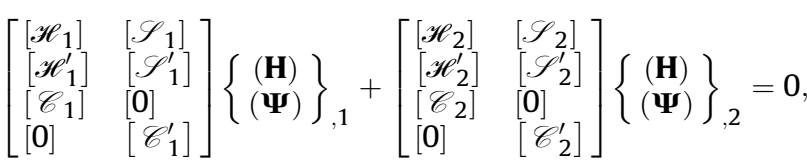

where

$$
\begin{aligned}
& {\left[\mathscr{H}_{1}\right]=\left[\begin{array}{ll}
1 & 0
\end{array}\right]+\left[\begin{array}{ll}
1 & 0
\end{array}\right]\left[\mathbb{K}^{\prime}\right], \quad\left[\mathscr{S}_{1}\right]=\left[\begin{array}{ll}
1 & 0
\end{array}\right]\left[\mathbb{L}^{\prime}\right][\mathscr{E}],} \\
& {\left[\mathscr{H}_{2}\right]=\left[\begin{array}{ll}
0 & 1
\end{array}\right]+\left[\begin{array}{ll}
0 & 1
\end{array}\right]\left[\mathbb{K}^{\prime}\right], \quad\left[\mathscr{S}_{2}\right]=\left[\begin{array}{ll}
0 & 1
\end{array}\right]\left[\mathbb{L}^{\prime}\right][\mathscr{E}],} \\
& \begin{array}{l}
{\left[\mathscr{H}_{1}^{\prime}\right]=\mu_{0}\left[\begin{array}{ll}
H_{1} & 0 \\
H_{2} & 0
\end{array}\right]+\left[\begin{array}{lll}
1 & 0 & 0 \\
0 & 1 & 0
\end{array}\right][\mathbb{K}], \quad\left[\mathscr{S}_{1}^{\prime}\right]=\left[\begin{array}{lll}
1 & 0 & 0 \\
0 & 1 & 0
\end{array}\right][\mathbb{Z}][\mathscr{E}],} \\
{\left[\mathscr{H}_{2}^{\prime}\right]=\mu_{0}\left[\begin{array}{ll}
0 & H_{1} \\
0 & H_{2}
\end{array}\right]+\left[\begin{array}{lll}
0 & 1 & 0 \\
0 & 0 & 1
\end{array}\right][\mathbb{K}], \quad\left[\mathscr{S}_{2}^{\prime}\right]=\left[\begin{array}{lll}
0 & 1 & 0 \\
0 & 0 & 1
\end{array}\right][\mathbb{[}][\mathscr{E}],}
\end{array} \\
& {\left[\mathscr{C}_{1}\right]=\left[\begin{array}{ll}
0 & 1
\end{array}\right], \quad\left[\begin{array}{l}
\mathscr{C}_{1}^{\prime} \\
]
\end{array}\right]=\left[\begin{array}{cccc}
0 & 0 & -1 & 0 \\
0 & -1 & 0 & 0
\end{array}\right] \text {, }} \\
& {\left[\mathscr{C}_{1}\right]=\left[\begin{array}{ll}
-1 & 0
\end{array}\right], \quad\left[\mathscr{C}_{1}^{\prime}\right]=\left[\begin{array}{llll}
1 & 0 & 0 & 0 \\
0 & 0 & 0 & 1
\end{array}\right] \text {. }}
\end{aligned}
$$

The detailed derivation can be found in A. We denote the above system of equations in the following compact form,

$\mathbf{A} \Theta_{, 1}+\mathbf{B} \Theta_{, 2}=0$,

where $\Theta=\left\{H_{1}, H_{2}, \Psi_{11}, \Psi_{22}, \Psi_{12}, \Psi_{21}\right\}^{T}$. The above system becomes elliptic, parabolic or hyperbolic when the eigenvalues of the following characteristic equation are complex, equal or real, respectively

$\operatorname{det}(\mathbf{B}-\alpha \mathbf{A})=0$,

with $\alpha$ being the eigenvalue of the system.

\section{Analytic results of stability analysis}

.Our stability analysis will be based on the magnetization responses in Fig. 3, where the newly developed model predictions are compared with Kiefer-Lagoudas model (Kiefer and Lagoudas, 2005). ${ }^{2}$ The most important material constants for the model are identified as $M^{\text {sat }}, K, H^{C}, H_{s}^{M_{2}}, H_{f}^{M_{2}}, H_{s}^{M_{1}}, H_{f}^{M_{1}}, \mathbb{E}, \varepsilon^{\max }, \sigma^{*}$ and $n_{1}, n_{2}, n_{3}$, $n_{4}$ (Table B.7). ${ }^{3}$ We consider $M^{\text {sat }}, H^{C}$ and $\mathbb{E}$ to non-dimensionalize the remaining material constants and material parameters. The required material parameters are presented in Table 1 . The nondimensionalized material constants and material parameters are given in Tables 2 and 3, respectively. In terms of space nondimensionalization, we consider $\widehat{x}_{1}=x_{1} / w, \widehat{x}_{2}=x_{2} / l, w / l=\gamma$, where $w$ is the width and $l$ is the length of the specimen. Magnetic field is non-dimensionalized as $\widehat{H}_{1}=\frac{\beta H_{1}}{M^{\text {sat }}}$ and $\widehat{H}_{2}=\frac{\beta H_{2}}{M^{\text {sat }}}$. Moreover we define non-dimensional quantities $\lambda=\frac{\mu_{0}\left(M^{\text {sat }}\right)^{2}}{\mathbb{E}}, \widehat{r}=\frac{r}{\mathbb{F}}$ and $\widehat{\sigma}^{*}=\frac{\sigma^{*}}{\mathbb{E}}$, where $r=\frac{\partial \Phi}{\partial \xi}$.

Three case studies for stability conditions will be discussed next. First (Case-I) we only consider the magnetostatic problem for isotropy. Next we consider the coupled system with isotropy (CaseII) and finally (Case-III) the anisotropic effect on the stability for the coupled system is considered.

\subsection{Case-I: magnetostatic stability condition}

We start stability analysis with a simple magnetostatic case.

\footnotetext{
2 The values of the material constants can be found in (Haldar et al., 2011).

${ }^{3}$ A brief description of parameter identification procedure is presented in Appendix B.
} 
6.1.1. Before reorientation

Considering magnetostatic equations before reorientation, we can write Eq. (47) in a non-dimensional way as

$\gamma\left[\begin{array}{cc}1 & \beta \frac{\partial \widehat{a}_{1}}{\partial \widehat{H}_{2}} \\ 0 & -1\end{array}\right]\left(\begin{array}{l}\widehat{H}_{1} \\ \widehat{H}_{2}\end{array}\right), \widehat{1}^{1}+\left[\begin{array}{cc}0 & (1+\beta) \\ 1 & 0\end{array}\right]\left(\begin{array}{l}\widehat{H}_{1} \\ \widehat{H}_{2}\end{array}\right)_{, \widehat{2}}=0$.

The characteristic polynomial Eq. (48) is given by

$\alpha^{2}-\beta \frac{\partial \widehat{a}_{1}}{\partial \widehat{H}_{2}} \alpha+(1+\beta)=0$

for which the discriminant is

$\widehat{D}:=\frac{(\beta+2)^{2} \widehat{H}_{2}^{2}-4(1+\beta)}{1-\widehat{H}_{2}^{2}}$.

Since $\widehat{H}_{2}=\frac{\beta H_{2}}{M^{\text {sat }}}=\frac{H_{2}}{H^{C}} \widehat{M}_{y}^{C}$ and $\widehat{M}_{y}^{C}<1$, for $H_{2} \in\left[0, H^{C}\right], 1-\widehat{H}_{2}^{2}>0$. Thus the sign of $\widehat{D}$ will depend on the numerator only. The numerator $(\mathrm{Nr})$ further can be written as

$N r=4(1+\beta)\left[\frac{(\beta+2)^{2}}{4(1+\beta)} \widehat{H}_{2}^{2}-1\right]$.

At $H_{2}=\widehat{H}_{2}=0, \widehat{D}<0$ since for this particular problem $\beta=0.776>0$. Similarly, at $H_{2}=H^{C}, \widehat{H}_{2} \approx 0.5$ (see Fig. 3), and again $\widehat{D}<0$. So the system is elliptic in the entire range $\left[0, H^{C}\right]$.

\subsubsection{During reorientation}

When reorientation starts, from (47) we can write

$$
\left.\gamma\left[\begin{array}{cc}
1 & \frac{\widehat{M}_{x}^{C} \Delta \widehat{M} \lambda}{\widehat{r}} \\
0 & 1
\end{array}\right]\left(\begin{array}{l}
\widehat{H}_{1} \\
\widehat{H}_{2}
\end{array}\right)_{, \hat{1}}+\left[\begin{array}{cc}
0 & 1-\frac{\Delta \widehat{M}^{2} \lambda}{\widehat{r}}
\end{array}\right)\right]\left(\begin{array}{c}
\widehat{H}_{1} \\
\widehat{H}_{2}
\end{array}\right)_{, 2}=0 .
$$

The characteristic polynomial (48) is given by

$$
\left.\alpha^{2}-\frac{\widehat{M}_{\chi}^{C} \Delta \widehat{M} \lambda}{\widehat{r}} \alpha+1-\frac{\Delta \widehat{M}^{2} \lambda}{\widehat{r}}\right)=0
$$

and so the discriminant

$$
\widehat{D}:=\left[\left(\frac{\widehat{M}_{\chi}^{C} \Delta \widehat{M} \lambda}{\widehat{r}}\right)^{2}+4 \frac{\Delta \widehat{M}^{2} \lambda}{\widehat{r}}\right]-4 .
$$

The elliptic/hyperbolic nature of the system depends on the sign of $\widehat{D}$. When $\widehat{D} \geq 0$, the system is hyperbolic and when $\widehat{D}<0$, the system is elliptic. Fig. 4 shows that the system behaves hyperbolic at the beginning but recovers ellipticity around $\widehat{H}_{2}=0.7$ or at 0.84 [T].

\subsection{Magneto-mechanical stability condition}

\subsubsection{Case-II: isotropic medium}

As a next step, we consider a coupled magneto-mechanical stability analysis by assuming an isotropic medium. The system of equations can then be written as

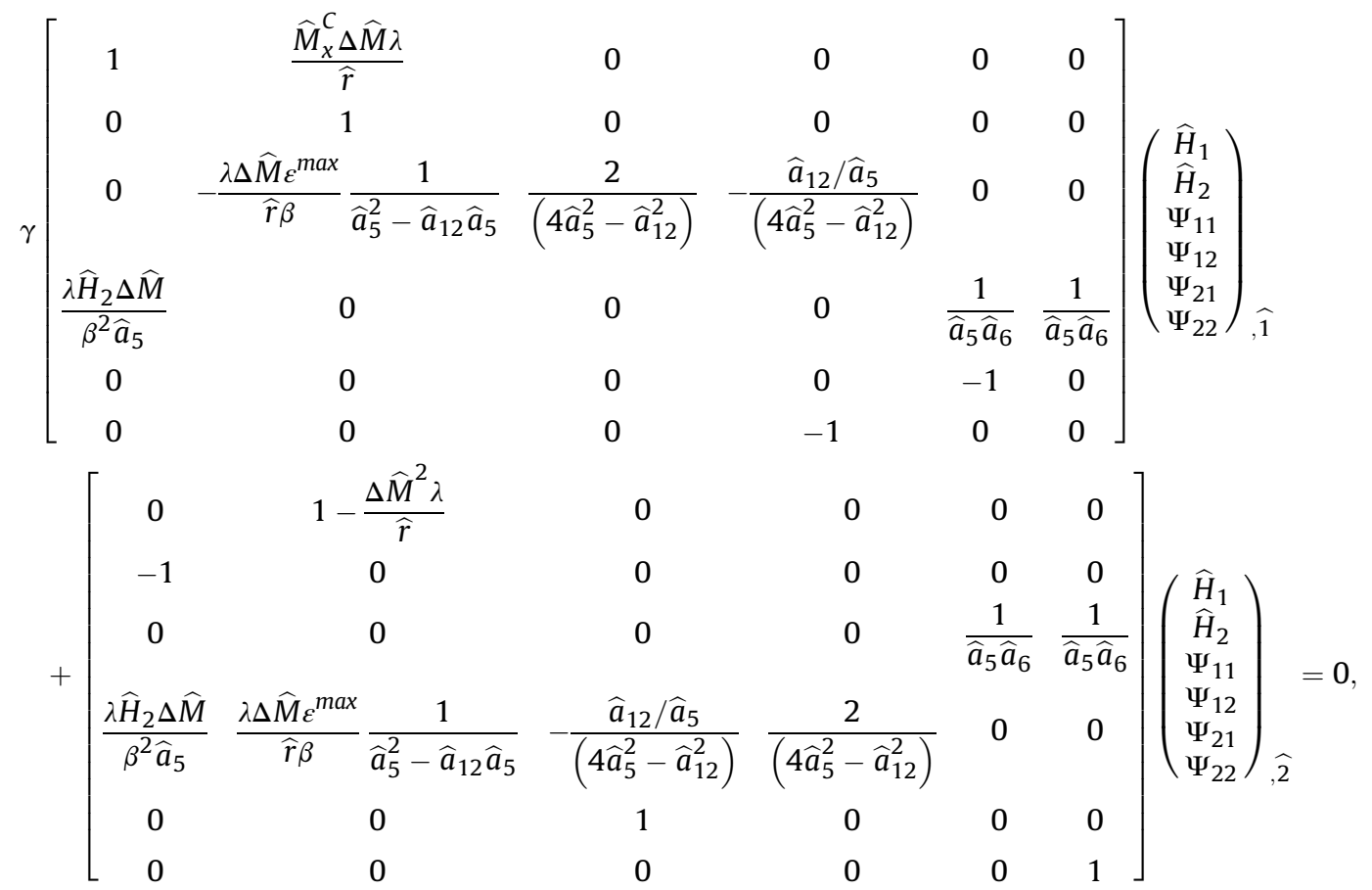




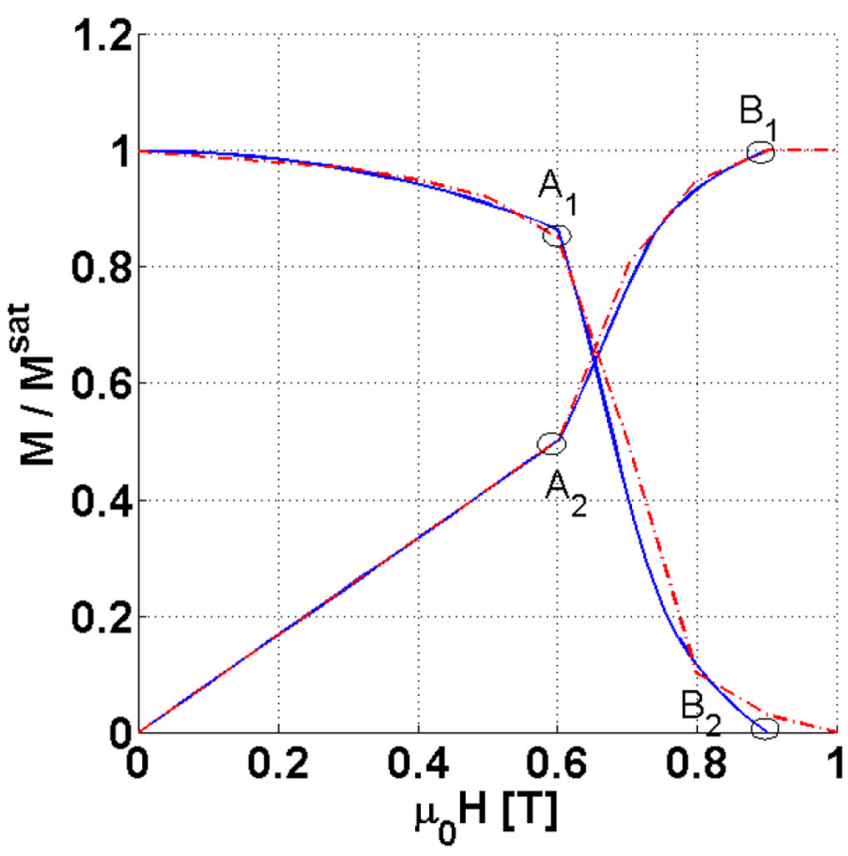

Fig. 3. Model prediction comparison of magnetization responses with Kiefer-Lagoudas model (red dotted, with demagnetization correction Haldar et al. (2011)) and present model (blue continuous). $A_{2} B_{1}$ is the y-component and $A_{1} B_{2}$ is the x-component. (For interpretation of the references to colour in this figure legend, the reader is referred to the web version of this article.)

Table 1

Material parameters (isotropic medium).

$$
\begin{aligned}
& M_{y}^{C}=\mu_{0}\left(K M^{\text {sat }}\right) H_{s}^{M_{2}}, M_{x}^{C}=\sqrt{\left(M^{\text {sat }}\right)^{2}-\left(M_{y}^{C}\right)^{2}} \\
& 2 a_{3}=\mu_{0} \beta, \beta=\mu_{0} K M^{\text {sat }}, b_{1}=\mu_{0} M^{\text {sat }}, \Delta M=M^{\text {sat }}-M^{C} \\
& a_{1}=\mu_{0} \sqrt{\left(M^{\text {sat }}\right)^{2}-\beta^{2} H_{2}^{2}}, \forall H_{2} \in\left[0, H^{C}\right), \text { and } \\
& a_{1}=\mu_{0} M_{x}^{C}, \forall H_{2} \in\left[H^{C}, H_{f}^{M_{2}}\right] \\
& a_{5}=a_{4}=\frac{1}{2 \mathbb{E}}, a_{12}=\frac{-\nu}{\mathbb{E}}, a_{6}=\frac{1+\nu}{2 \mathbb{E}}, b_{10}=d_{4}=0, \tilde{d}=\varepsilon^{\max }
\end{aligned}
$$

for which the characteristic equation is

$\left.\left[\alpha^{2}-\frac{\widehat{M}_{\chi}^{C} \Delta \widehat{M} \lambda}{\widehat{r}} \alpha+1-\frac{\Delta \widehat{M}^{2} \lambda}{\widehat{r}}\right)\right]\left[\alpha^{2}+1\right]^{2}=0$.

For the real root, we only need to consider

$\left.\alpha^{2}-\frac{\widehat{M}_{\chi}^{C} \Delta \widehat{M} \lambda}{\widehat{r}} \alpha+1-\frac{\Delta \widehat{M}^{2} \lambda}{\widehat{r}}\right)=0$

and so the conditions of stability are the same as for the magnetostatic (Case-I). It should be noted that the nonlinear magnetization constitutive equation causes the unstable behavior to the entire magneto-mechanical system.

\subsubsection{Case-III: anisotropic medium}

This part is the main application of an anisotropic magnetomechanical coupling. Since, we do not have anisotropic magneto-

Table 2

Non-dimensionalized material constants.

$\widehat{H}_{s}^{M_{2}}=1, \widehat{H}_{f}^{M_{2}}=\frac{H_{f}^{M_{2}}}{H^{\mathrm{C}}}, \widehat{H}_{s}^{M_{1}}=\frac{H_{s}^{M_{1}}}{H^{\mathrm{C}}}, \widehat{H}_{f}^{M_{1}}=\frac{H_{f}^{M_{1}}}{H^{\mathrm{C}}}$
Table 3

Non-dimensionalized material parameters (isotropic medium).

\begin{tabular}{l}
$\widehat{M}_{y}^{C}=\frac{M_{y}^{c}}{M^{\text {sat }}}=\mu_{0} K H^{C}, \widehat{M}_{x}^{C}=\frac{M_{x}^{c}}{M^{\text {sat }}}=\sqrt{1-\left(\widehat{M}_{y}^{C}\right)^{2}}$ \\
$2 \widehat{a}_{3}=\frac{2 a_{3}}{\mu_{0}}=\beta, \widehat{b}_{1}=\frac{b_{1}}{\mu_{0} M^{\text {sat }}}=1, \Delta \widehat{M}=\frac{\Delta M}{M^{\text {sat }}}$ \\
$\widehat{a}_{1}=\frac{a_{1}}{\mu_{0} M^{\text {sat }}}=\sqrt{1-\widehat{H}_{2}^{2}}, \forall H_{2} \in\left[0, H^{C}\right)$, and \\
$\widehat{a}_{1}=\frac{a_{1}}{\mu_{0} M^{\text {sat }}}=\widehat{M}_{x}^{C}, \forall H_{2} \in\left[H^{C}, H_{f}^{M}\right], p=0$ \\
$\widehat{a}_{5}=\widehat{a}_{4}=\frac{1}{2}, \widehat{a}_{12}=-\nu, \widehat{a}_{6}=\frac{1+v}{2}, \tilde{d}=\varepsilon^{\max }$ \\
$\lambda=\frac{\mu_{0}\left(M^{\text {sat }}\right)^{2}}{\mathbb{E}}, \widehat{r}=\frac{r}{\mathbb{E}}, \widehat{\sigma}^{*}=\frac{\sigma^{*}}{\mathbb{E}}$ \\
\hline
\end{tabular}

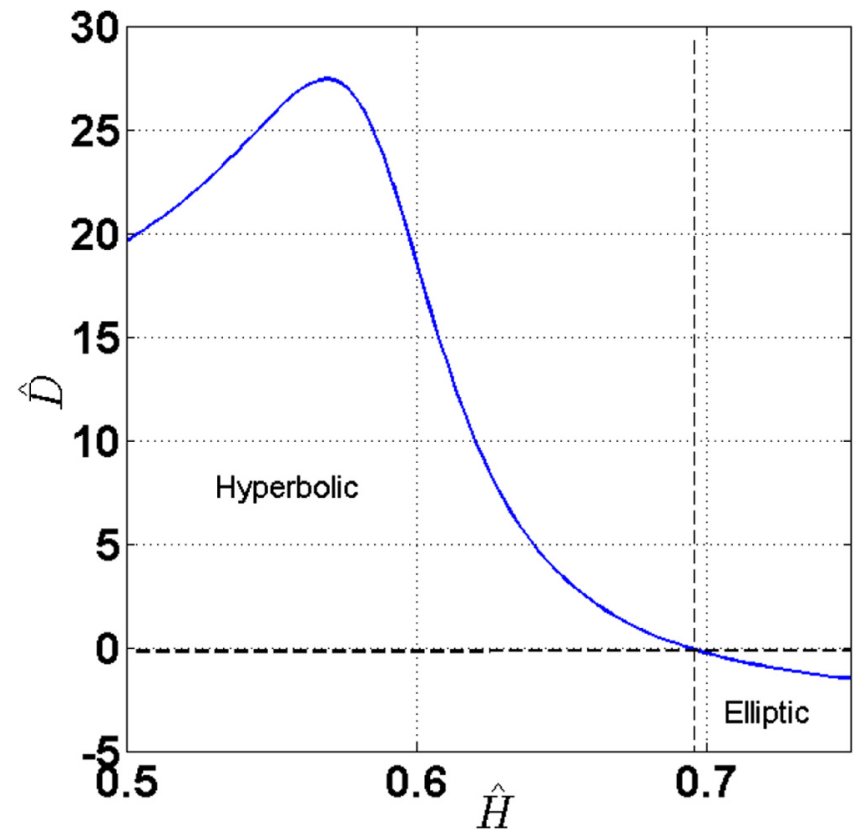

Fig. 4. Change in sign of $\widehat{D}$ during the reorientation process.

mechanical experimental characterization for the model calibration, we only assume the following (from (33), (34)) non zero material constants for simplicity:

$$
\left(\begin{array}{c}
\varepsilon_{11}^{1} \\
\varepsilon_{12}^{1} \\
\varepsilon_{22}^{1}
\end{array}\right)=\left[\begin{array}{ccc}
2 a_{5} & 0 & a_{12} \\
0 & 0 & 2 a_{6} \\
a_{12} & 0 & 2 a_{4}
\end{array}\right]\left(\begin{array}{c}
\sigma_{11}^{E} \\
\sigma_{12}^{E} \\
\sigma_{22}^{E}
\end{array}\right)
$$

$$
\left(\begin{array}{c}
\varepsilon_{11}^{2} \\
\varepsilon_{12}^{2} \\
\varepsilon_{22}^{2}
\end{array}\right)=\left[\begin{array}{ccc}
2 b_{4} & 0 & b_{12} \\
0 & 0 & 2 b_{6} \\
b_{12} & 0 & 2 b_{5}
\end{array}\right]\left(\begin{array}{c}
\sigma_{11}^{E} \\
\sigma_{12}^{E} \\
\sigma_{22}^{E}
\end{array}\right)+\left[\begin{array}{cc}
0 & b_{10} \\
0 & 0 \\
0 & 0
\end{array}\right]\left(\begin{array}{c}
H_{1} \\
H_{2}
\end{array}\right) .
$$

Recall that superscripts 1 and 2 are for the variant- 1 and variant2 respectively. The coefficient $b_{10}$ couples $\mathrm{H}_{2}$ with $\varepsilon_{11}^{2}$, i.e, a piezomagnetic coefficient for variant-2. Since both the variants have the same tetragonal structure, one needs to satisfy that the Gibbs free energy $G^{V_{1}}=G^{V_{2}}$. We can thus immediately write $b_{4}=a_{5}, b_{12}=a_{12}$, $b_{5}=a_{4}$ and $b_{6}=a_{6}$ for purely mechanical conditions (from (9) and (12)). 


$$
\left.\begin{array}{l}
M_{1}^{1} \\
M_{2}^{1}
\end{array}\right)=\left(\begin{array}{c}
\tilde{a}_{1} \\
0
\end{array}\right)
$$

and

$$
\left.\begin{array}{l}
M_{1}^{2} \\
M_{2}^{2}
\end{array}\right)=\left[\begin{array}{ccc}
0 & 0 & 0 \\
b_{10} / \mu_{0} & 0 & 0
\end{array}\right]\left(\begin{array}{c}
\sigma_{11}^{E} \\
\sigma_{12}^{E} \\
\sigma_{22}^{E}
\end{array}\right)+\left(\begin{array}{c}
0 \\
b_{1} / \mu_{0}
\end{array}\right) .
$$

The additional material constants for the anisotropic medium is summarized in Table 4 and the non-dimensionalization is presented in Table 5. Moreover, like isotropy, we consider $\widehat{r}=\frac{r}{F_{1}}$, $\lambda=\frac{\mu_{0}\left(M^{\text {sat }}\right)^{2}}{\mathbb{E}_{1}}$ and $\widehat{\sigma}^{*}=\frac{\sigma^{*}}{\mathbb{E}_{1}}$. The non-dimensionalized system of PDEs can then be written as
Table 4

Material constants (anisotropy).

$a_{5}=\frac{1}{2 \mathbb{E}_{1}}, a_{4}=\frac{1}{2 \mathbb{E}_{2}}, a_{12}=\frac{-\nu_{12}}{\mathbb{E}_{1}}=\frac{-\nu_{21}}{\mathbb{E}_{2}}, a_{6}=\frac{1+\nu_{12}}{\mathbb{E}_{1}}, b_{10}$

Table 5

Material parameters (anisotropy).

$\widehat{a}_{5}=\frac{1}{2}, \widehat{a}_{4}=\frac{\mathbb{E}_{1}}{2 \mathbb{E}_{2}}, \widehat{a}_{12}=-\nu_{12}=\frac{-\nu_{21} \mathbb{E}_{1}}{\mathbb{E}_{2}}, \widehat{a}_{6}=1+\nu_{12}, \widehat{b}_{10}=\frac{M^{\text {sat }} b_{10}}{\beta}$

$\widehat{r}=\frac{r}{\mathbb{E}_{1}}, \lambda=\frac{\mu_{0}\left(M^{\text {sat }}\right)^{2}}{\mathbb{E}_{1}}, \widehat{\sigma}^{*}=\frac{\sigma^{*}}{\mathbb{E}_{1}}$

$\gamma\left[\begin{array}{cccccc}1 & \frac{\widehat{M}_{x}^{C} \Delta \widehat{M} \lambda}{\widehat{r}} & 0 & 0 & 0 & 0 \\ 0 & 1 & 0 & 0 & 0 & 0 \\ 0 & \widehat{A}_{32} & 2 & -\frac{\widehat{a}_{12}}{\widehat{a}_{4}} & 0 & 0 \\ \frac{\lambda \widehat{H}_{2} \Delta \widehat{M}}{\beta^{2} \widehat{C} \widehat{a}_{4}} & 0 & 0 & 0 & \frac{1}{\widehat{\mathbb{C}} \widehat{a}_{4} \widehat{a}_{6}} & \frac{1}{\widehat{C} \widehat{a}_{4} \widehat{a}_{6}} \\ 0 & 0 & 0 & 0 & -1 & 0 \\ 0 & 0 & 0 & -1 & 0 & 0\end{array}\right]\left(\begin{array}{c}\widehat{H}_{1} \\ \widehat{H}_{2} \\ \Psi_{11} \\ \Psi_{12} \\ \Psi_{21} \\ \Psi_{22}\end{array}\right)_{, 1}$

$+\left[\begin{array}{cccccc}0 & \widehat{B}_{12} & \frac{2 \widehat{a}_{4} \widehat{b}_{10} \xi \widehat{\mathbb{C}} \beta^{2}}{\lambda} & \frac{-\widehat{a}_{12} \widehat{b}_{10} \xi \widehat{\mathbb{C}} \beta^{2}}{\lambda} & 0 & 0 \\ -1 & 0 & 0 & 0 & 0 & 0 \\ 0 & 0 & 0 & 0 & \frac{1}{\widehat{C} \widehat{a}_{4} \widehat{a}_{6}} & \frac{1}{\widehat{\mathbb{C}} \widehat{a}_{4} \widehat{a}_{6}} \\ \frac{\lambda \widehat{H}_{2} \Delta \widehat{M}}{\beta^{2} \widehat{\mathbb{C}}_{4}} & \widehat{B}_{42} & -\frac{\widehat{a}_{12}}{\widehat{a}_{4}} & \frac{2 \widehat{a}_{5}}{\widehat{a}_{4}} & 0 & 0 \\ 0 & 0 & 1 & 0 & 0 & 0 \\ 0 & 0 & 0 & 0 & 0 & 1\end{array}\right]\left(\begin{array}{c}\widehat{H}_{1} \\ \widehat{H}_{2} \\ \Psi_{11} \\ \Psi_{12} \\ \Psi_{21} \\ \Psi_{22}\end{array}\right)_{, \widehat{2}}=0$,

where,

$$
\begin{aligned}
\widehat{\mathbb{C}}= & \frac{1}{4 \widehat{a}_{4} \widehat{a}_{5}-\widehat{a}_{12}^{2}} \\
\widehat{A}_{32}= & \frac{\lambda \Delta \widehat{M}}{\widehat{r}}\left(\frac{\widehat{a}_{12}}{\widehat{a}_{4}} \varepsilon^{\max }+2\left(\varepsilon^{\max }+\widehat{H}_{2} \widehat{b}_{10}\right)\right)-2 \widehat{b}_{10} \xi \\
\widehat{B}_{12}= & 1-\frac{\widehat{\mathbb{C}} \widehat{a}_{4} \widehat{b}_{10}^{2} \beta^{2} \xi^{2}}{\lambda}-\frac{\Delta \widehat{M}^{2} \lambda}{\widehat{r}}+\beta \frac{\widehat{\sigma}^{*} \widehat{b}_{10}}{\widehat{r}} \\
& \left.+\beta \frac{\widehat{b}_{10} \widehat{\mathbb{C}}}{\widehat{r}}\left(\widehat{a}_{12} \varepsilon^{\max } \xi+2 \widehat{a}_{4}\left(\varepsilon^{\max }+\widehat{H}_{2} \widehat{b}_{10}\right)\right)\right) \\
\widehat{B}_{42}= & \frac{\Delta \widehat{M} \lambda}{\widehat{r}}\left(2 \frac{\widehat{a}_{5}}{\widehat{a}_{4}} \varepsilon^{\max }+\frac{\widehat{a}_{12}}{\widehat{a}_{4}}\left(\varepsilon^{\max }+\widehat{H}_{2} \widehat{b}_{10}\right)\right)+\frac{\widehat{a}_{12}}{\widehat{a}_{4}} \widehat{b}_{10} \xi
\end{aligned}
$$

and the characteristic polynomial is in the following form

$t_{6} \alpha^{6}+t_{5} \alpha^{5}+t_{4} \alpha^{4}+t_{3} \alpha^{3}+t_{2} \alpha^{2}+t_{1} \alpha+t_{0}=0$

where the coefficients are

$$
\begin{aligned}
t_{6}= & 1 t_{5}=-\frac{\widehat{M}_{x}^{C} \Delta \widehat{M} \lambda}{\widehat{r}} t_{4} \\
= & \left(1+\frac{2 \widehat{a}_{6}}{\widehat{a}_{4}}+\frac{\widehat{a}_{12}}{\widehat{a}_{4}}-\widehat{H}_{2} \widehat{b}_{10} \xi\right)-\frac{\Delta \widehat{M}}{\widehat{r}}\left(\Delta \widehat{M} \lambda-\widehat{\sigma}^{*} \widehat{b}_{10} \beta\right) t_{3} \\
= & -\widehat{H}_{2} \widehat{b}_{10} \xi-\frac{\Delta \widehat{M}_{\bar{M}}^{C} \lambda}{\widehat{r}}\left(2 \frac{\widehat{a}_{6}}{\widehat{a}_{4}}+\frac{\widehat{a}_{12}}{\widehat{a}_{4}}\right) t_{2} \\
= & \left.\frac{\widehat{a}_{5}}{\widehat{a}_{4}}+\widehat{H}_{2} \widehat{b}_{10} \xi \frac{\widehat{a}_{12}}{2 \widehat{a}_{4}}+\frac{\widehat{a}_{6}+\widehat{a}_{12}}{\widehat{a}_{4}}\left[1+\frac{\Delta \widehat{M}^{2} \lambda}{\widehat{r}}+\beta \frac{\widehat{\sigma}^{*} \widehat{b}_{10}}{\widehat{r}}\right] t_{1}\right] \\
= & \widehat{a}_{5} \frac{\widehat{M}_{x}^{C} \Delta \widehat{M}_{\lambda} \lambda}{\widehat{a}_{4} \widehat{r}}-\frac{\widehat{H}_{2} \widehat{b}_{10} \widehat{a}_{12}}{2 \widehat{a}_{4}} \xi t_{0} \\
= & \left(\frac{\widehat{a}_{5}}{\widehat{a}_{4}}-\frac{\widehat{b}_{10}^{2} \beta^{2}}{\lambda \widehat{a}_{4}}\right)-\frac{\widehat{a}_{5} \Delta \widehat{M}^{2} \lambda}{\widehat{a}_{4} \widehat{r}}-\frac{\widehat{\sigma}^{*} \widehat{a}_{5} \Delta \widehat{M} \widehat{b}_{10} \beta}{\widehat{a}_{4} \widehat{r}} \\
& -\frac{1}{2} \Delta \widehat{M}_{10} \beta \widehat{b}^{\max } \xi-\frac{\Delta \widehat{M} \widehat{H}_{2} \widehat{b}_{10}^{2} \beta}{\widehat{a}_{4} \widehat{r}^{2}}
\end{aligned}
$$

Due to complex dependency of the polynomial coefficients on the material parameters, unlike previous two cases, it will be 
practically a tedious task to obtain the discriminant of a sextic equation. So, we like to analyze such a situation by numerically investigating the eigenvalues. If all the six eigenvalues are complex numbers, then the system will be elliptic, otherwise, with at least one real eigenvalue the loss of ellipticity will take place. We perform a parametric study with the piezomagnetic coefficient of variant- $2, b_{10}$, to investigate the influence of anisotropic coupling on the stability of the system. The result is shown in Fig. 5. We introduce a binary indicator I with a colormap such that when all the eigenvalues are complex numbers, $\mathrm{\imath}$ represents yellow and the system becomes elliptic. If at least one eigenvalue becomes real, it takes green color and loss of ellipticity occurs. As an example, in Fig. 5(a), for $\widehat{b}_{10}=1.0 \times 10^{-3}$, the material system becomes stable only after $\widehat{H} \approx 0.71$, where for $\widehat{b}_{10}>3.0 \times 10^{-3}$, the system remains unconditionally unstable. Note that the lower limit $\widehat{H}=0.5$ and the upper limit $\widehat{H}=0.75$ are the non-dimensionalized values of reorientation start field $\left(H_{s}^{M_{2}}\right)$ and reorientation finish field $\left(H_{f}^{M_{2}}\right)$. The entire scenario changes considerably by changing some material parameters in the magnetic constitutive equations (Fig. 3). Fig. 5(a) explains the effect of shifting the reorientation finish field $\left(H_{f}^{M_{2}}\right)$ as twice as the existing value. This means that in the latter case a less stiff constitutive equation is used, for which the material behaves more stable in the entire range of reorientation.

\section{Finite element analysis of the magneto-mechanically- coupled field equations for MSMA}

In the previous section we presented stability results for the non dimensionalized magneto-mechanical problem for MSMAs. All the analytical results were derived at a material point, based on the newly proposed constitutive responses. The results of the study show that the material instability may occur at a certain magnetic field or at a certain range of magnetic fields. The unstable behavior means that if a bulk material body is concerned and if there are singularities in the body, the disturbances due to the singularities propagate along the characteristics. This phenomenon is expected to be observed by solving an appropriate boundary value problem. We now consider a specific problem with real material parameters (Table 1) in the FE implementation. The value of the material constants (as presented in Table B.7) are taken from (Haldar et al., 2011).

Field equations, constitutive relations and boundary conditions are summarized in Table 6. Note that the magnetization constitutive equations depend only on the magnetic field and so do not

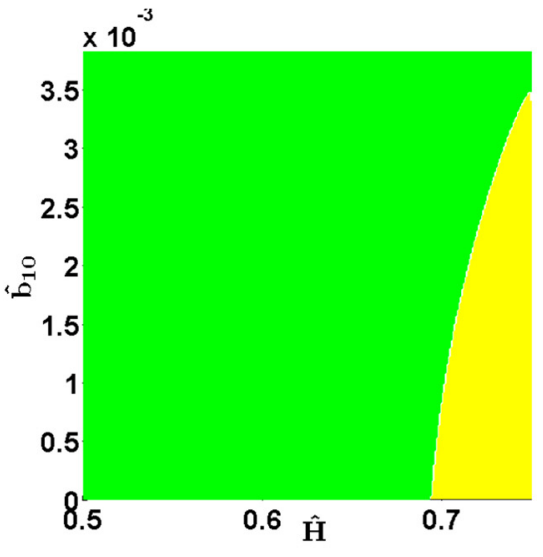

(a) account explicitly the influence of stress variation on the constitutive responses in the coupled problem. Moreover, the maximum strain $\varepsilon^{\max }$ is also assumed constant and so it is not coupled with the non-uniform stress distribution in the material.

The numerical analysis presented here was performed using the COMSOL Multi-physics finite element software package. The geometry and boundary conditions of the considered model problem are illustrated in Fig. 6. This particular arrangement is motivated by the experimental set up, explained in Appendix B. The computational domain may be regarded as the gap between the pole pieces of an electromagnet of dimensions $26 \mathrm{~mm} \times 26 \mathrm{~mm} \times 26 \mathrm{~mm}$ for which a uniform magnetic field of up to $2 \mathrm{~T}$ can be applied. Typical specimen dimensions are $8 \mathrm{~mm} \times 4 \mathrm{~mm} \times 4 \mathrm{~mm}$, or aspect ratios of $2: 1: 1$, where the long axis is the $x$-direction. All the field equations are summarized in Table 6 and more details can be found in Haldar et al. (2011). A spatially constant magnetic potential

$\Phi_{x}^{m}=\Phi_{y}^{m}=0 ; \quad \Phi_{z}^{m}=-\mu_{0} H_{y}^{a} x$

is applied on all sides of the boundary. The mechanical boundary conditions of the problem are illustrated in Fig. 7, where $t_{x}$ and $t_{y}$ denote the mechanical traction on the boundaries along the $x$ - and the $y$-directions, respectively. The compressive traction along the $x$ direction is imposed by constraining the vertical displacement $U$ of the $\partial \Omega_{3}$ surface and by applying a mechanical load $P=2 \mathrm{MPa}$ on the $\partial \Omega_{1}$ surface. We fix the point $R$ to eliminate rigid body motion in the finite element analysis.

\subsection{Numerical results}

Magnetic field $\left(\mathrm{H}_{2}\right)$ distribution at an applied field value $\mu_{0} H_{2}^{a}=$ 1.1 [T], for which the magnetostatic system becomes unstable, is presented in Fig. 8. It is numerically observed that two band-like zones are created inside the specimen. An enlarged view of the specimen is given in Fig. 9(a) with the plots of volume fraction $\xi$ of the newly oriented field induced variant (variant-2). In the figure, $\mu_{0}<H_{2}>=0.67[\mathrm{~T}]$ represents the true magnetic field inside the specimen in a constitutive sense (see, Fig. 3) corresponding to the applied field $\mu_{0} H_{2}^{a}=1.1[\mathrm{~T}]$ due to the shape and size effect of the specimen geometry. We observe that at the top-left and bottomright corners, the volume fraction almost reaches 1 while in the intermediate region, the volume fraction varies from 0 to 0.3 .

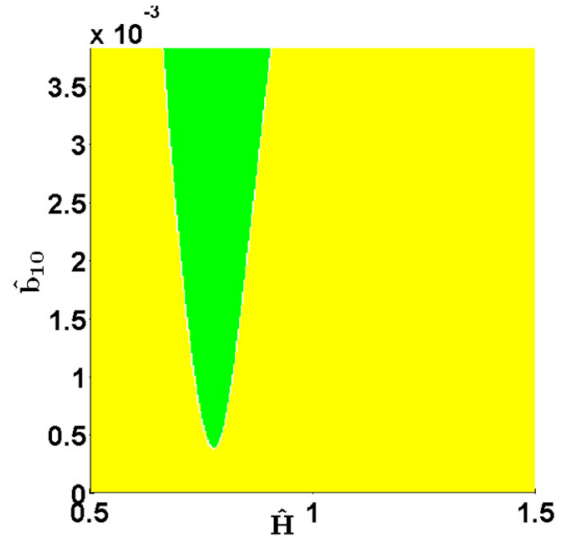

(b)



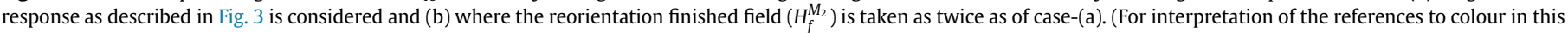
figure legend, the reader is referred to the web version of this article.) 
Table 6

Summary of the field equations, constitutive equations and boundary conditions.
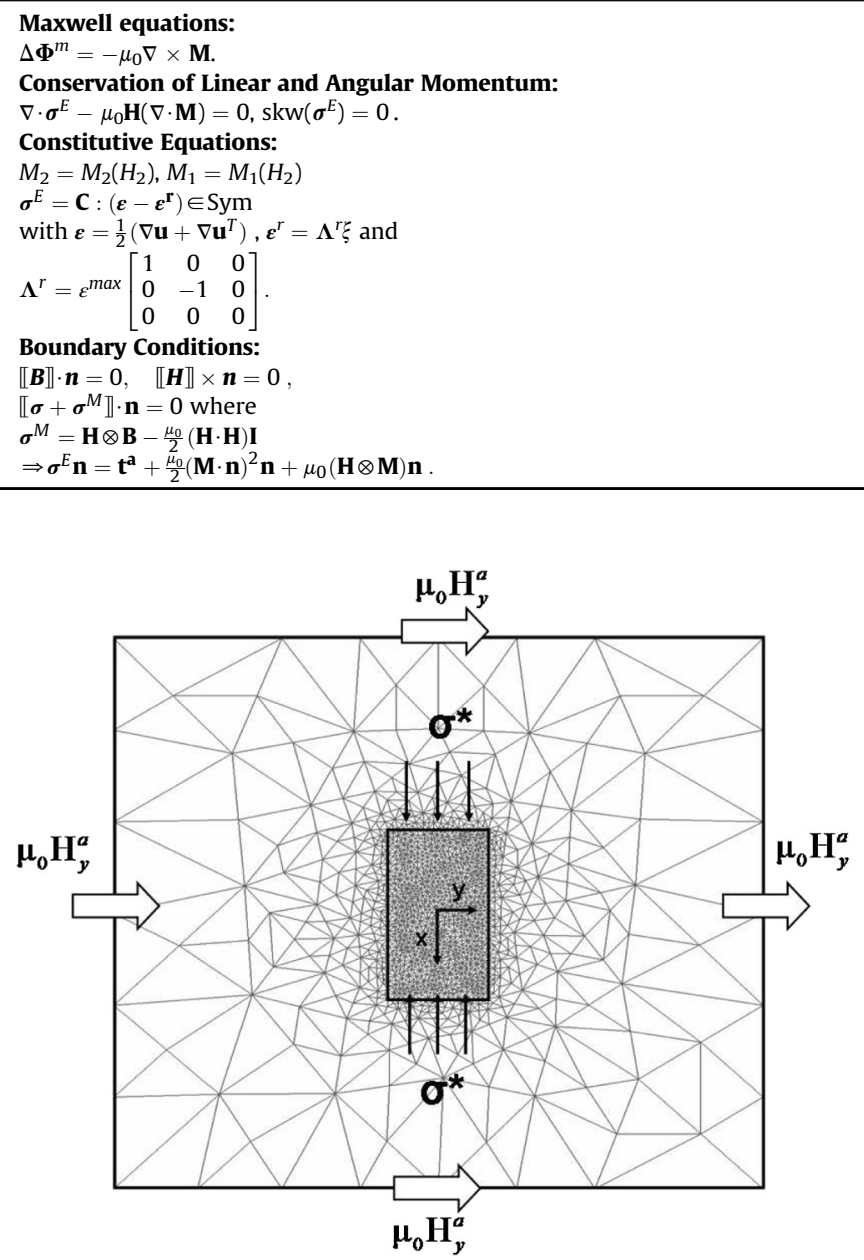

Fig. 6. Domain geometry, mesh and boundary conditions for the magnetostatic problem.

Moreover there are sharp changes in the values of $\xi$ across the observed banded zones. The magnetization vectors exhibit similar behavior in Fig. 9(b). The directions of magnetization vectors change very sharply in the regions $A_{1}-B_{1}-C_{1}-D_{1}$ and $A_{2}-B_{2}-C_{2}-D_{2}$. The appearance of the band like zones (Fig. 9) during reorientation is due to loss of ellipticity. The discriminant $D$ that dictates the loss of ellipticity is plotted at $\mu_{0}<H_{2}>=0.67$ [T] and is presented in Fig. 10(a). The Figure shows that for $\mu_{0}<H_{2}>=0.67$ [T] there are two distinct regions ' $H$ ' where $D \geq 0$ and loss of ellipticity occurs. The stable elliptic regions (' $E$ ' in Fig. 10(a)) with $D<0$, which are separated by the unstable hyperbolic regions, have a completely different behavior in terms of the field variables, like the magnetic field $\mathrm{H}_{2}$ (Fig. 10(b)), the martensitic variant volume fraction (Fig. 9(a)) and the magnetization vector (Fig. 9(b)).

The values of the characteristic angles in the unstable regions in the non-dimensional spatial description are given by the Eq. (57) and they vary spatially. In the present study the two characteristic angles of all the critical points are almost the same $\left(-60^{\circ}\right.$ and $-64^{\circ}$ in the actual specimen dimensions). The magnetic field shows a drastic change across characteristics that start from the top right and bottom left corners (Fig. 10(b)).

In the solution of strain (only $\varepsilon_{11}$ component is presented here) (Fig. 11(a)), we observe the banded regions. Similar trend is also

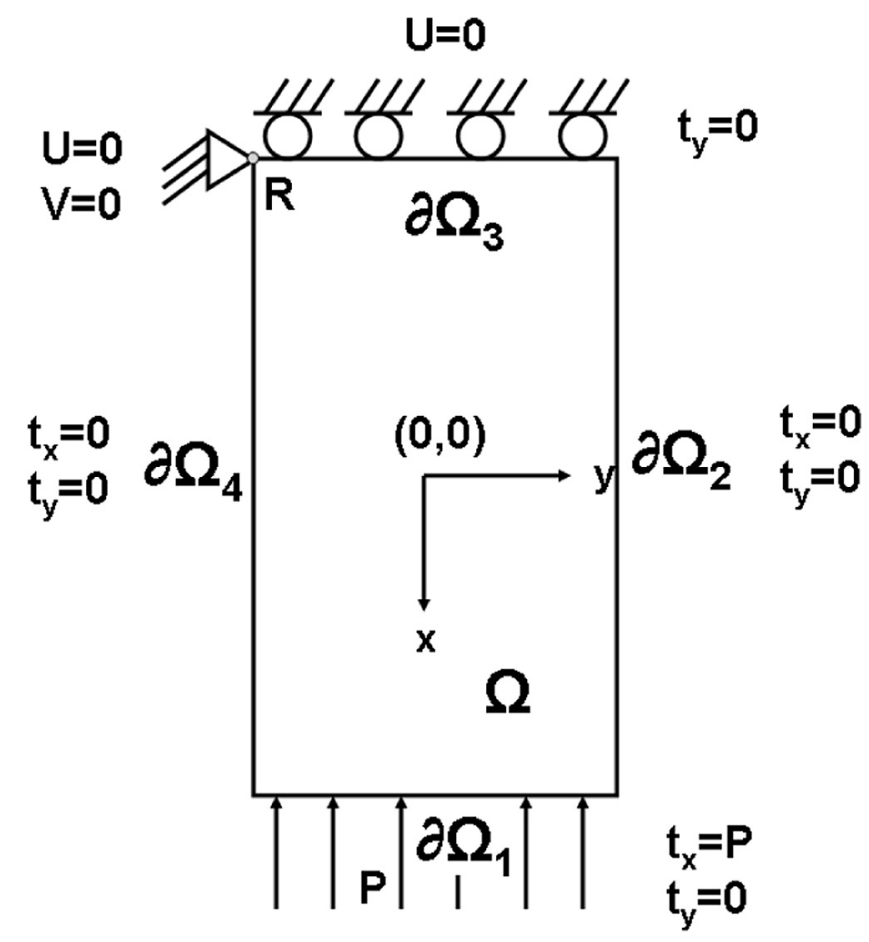

Fig. 7. Imposed mechanical boundary conditions. $\Omega$ is the material domain and $\partial \Omega$ its boundary.

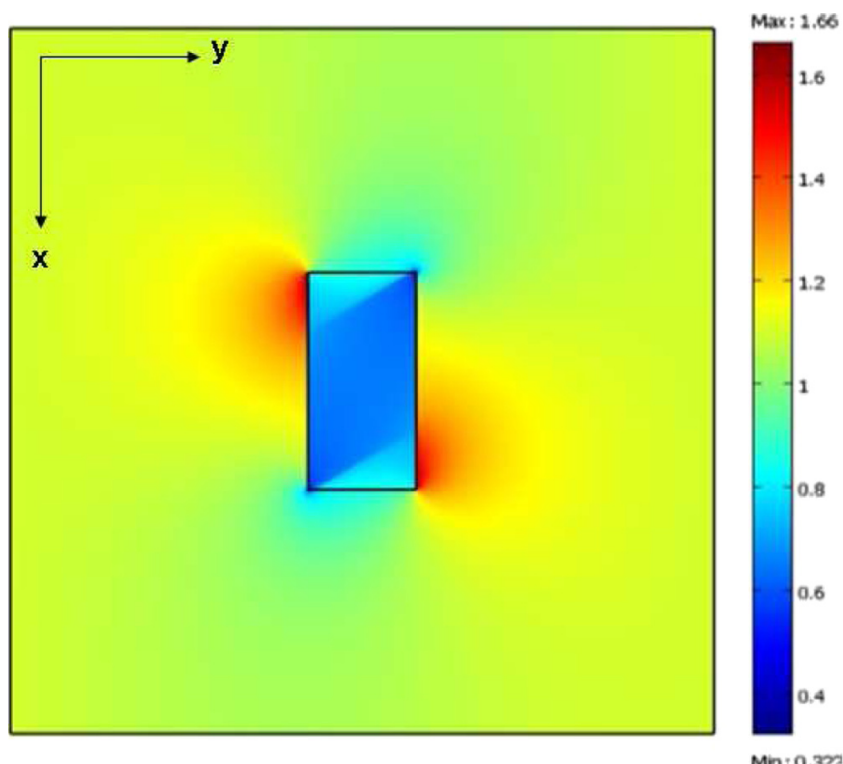

Fig. 8. Magnetic field distribution $\left(\mathrm{H}_{2}\right)$ inside and outside the specimen at the applied field $\mu_{0} H_{2}^{a}=1.1[\mathrm{~T}]$.

observed in the stress field ${ }^{4}$ (Fig. 11(b)). We like to emphasize here that the magnetization constitutive equation is assumed to depend only at a constant stress level. This means the variation of stress does not have any impact on the magnetic constitutive response. The main magneto-mechanical coupling in the system is through

\footnotetext{
${ }^{4}$ We plotted stress values only in the range 0-7 MPa. Level curves for higher values, which occur in particular near corners, are not shown.
} 


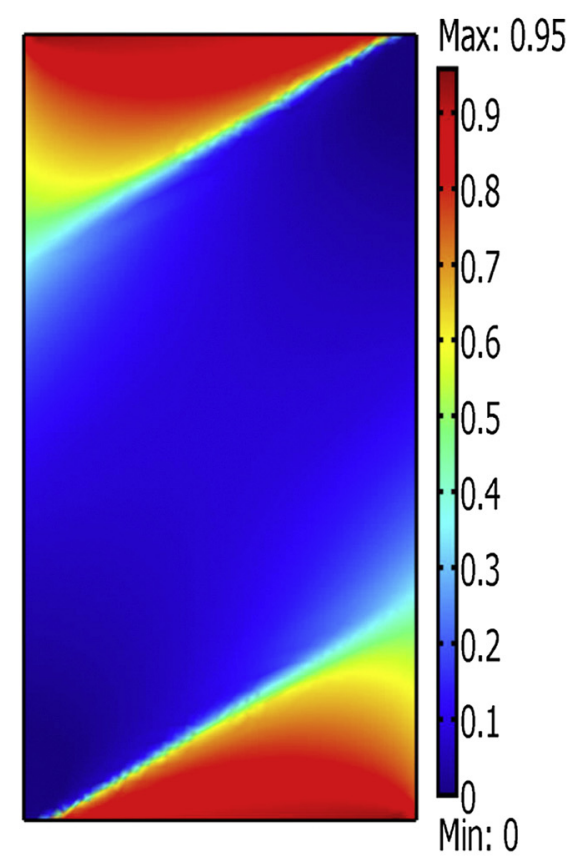

(a)



(b)

Fig. 9. (a) Volume fraction of field induced variant and (b) magnetic field $H_{2}$ at $\mu_{0}<H_{2}>=0.67$ [T].

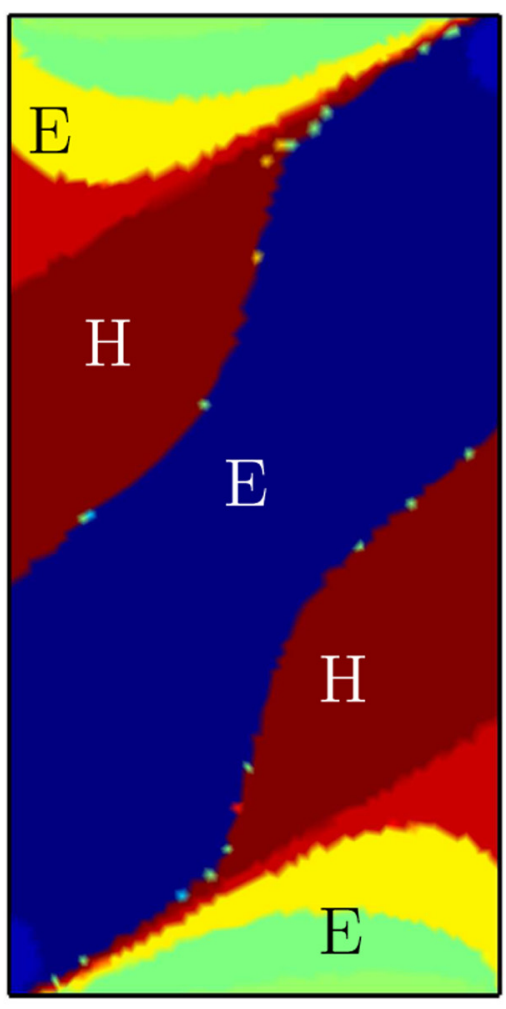

(a)

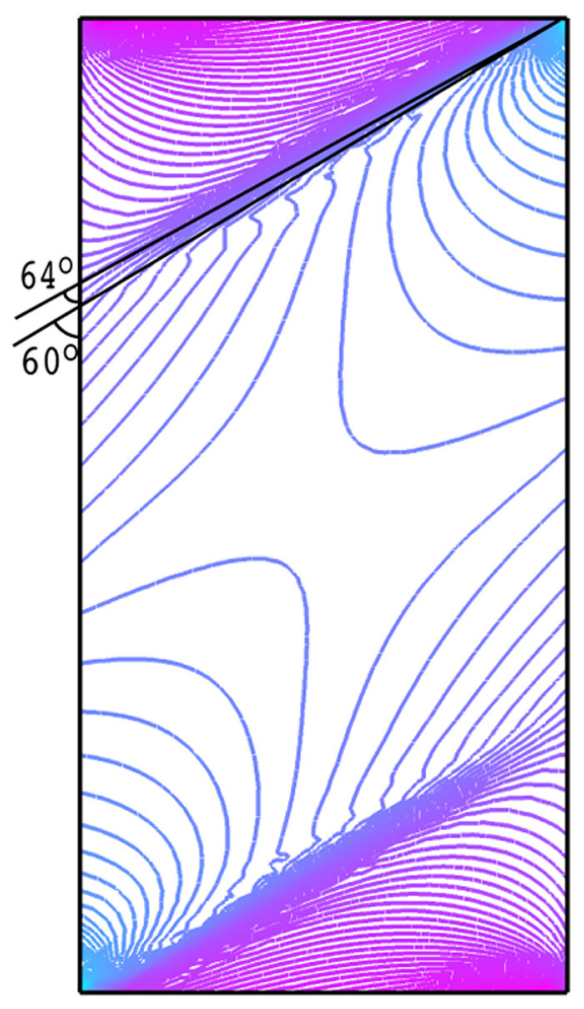

(b)

Fig. 10. (a) Discriminant $\mathrm{D}$ at $\mu_{0}<H_{2}>=0.67$ [T] and (b) jump in the magnetic field across characteristics. 


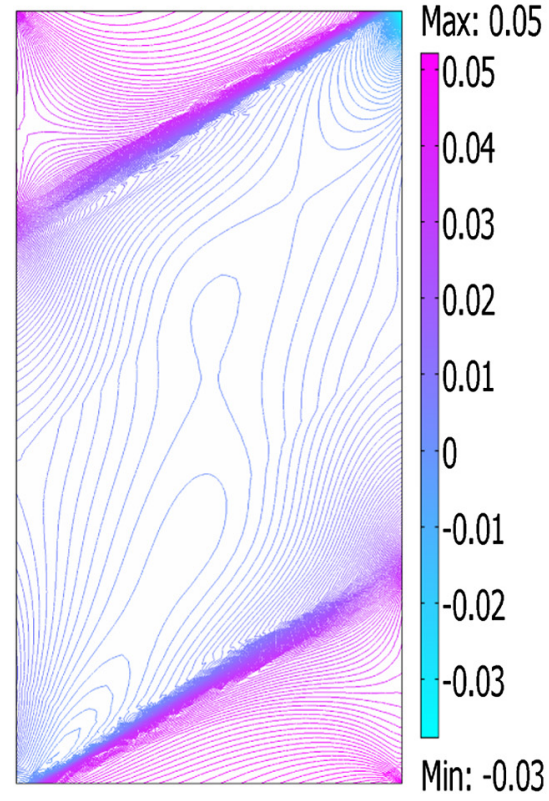

(a)

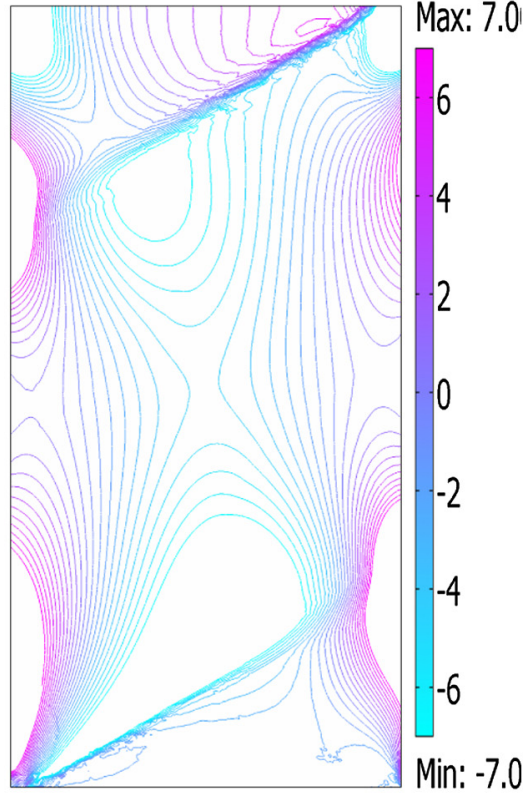

(b)

Fig. 11. Distribution of (a) $\varepsilon_{11}$ and (b) $\sigma_{11}$ at $\mu_{0}<H_{2}>=0.67$ [T](hyperbolic).



Max: 0.0555

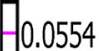

0.0554

0.0553

0.0553

0.0552

0.0552

0.0551

0.0551

0.055

0.0549

0.0549

0.0548

0.0548

0.0547

0.0547

0.0546

Min: 0.0546

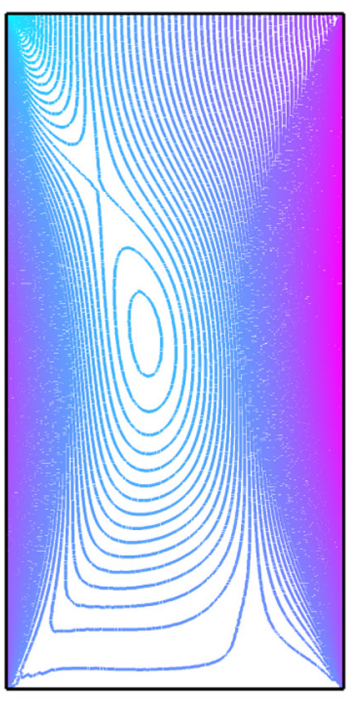

Max: -0.024

$-0.059$

$-0.302$

$-0.546$

$-0.789$

$-1.032$

$-1.275$

$-1.518$

$-1.762$

$-2.005$

$-2.248$

$-2.491$

$-2.734$

$-2.978$

$-3.221$

Min: -3.429

(a)

(b)

Fig. 12. Distribution of (a) $\varepsilon_{11}$ and (b) $\sigma_{11}$ at $\mu_{0}<H_{2}>=1$ [T] (elliptic).

the Maxwell equations, conservation of linear and angular momentum.

Finally, the system becomes elliptic everywhere at the end of reorientation and so becomes stable. The banded zones thus disappear from the solutions (Fig. 12).

\section{Discussion}

The coupled magneto-mechanical stability analysis shows that magnetic instabilities influences the mechanical field variables. The magnetic field singularities that occur at the corners of the specimen and propagate along the characteristics, intensifies the mechanical field variables in the banded regions. We observe around -7 MPa stress in the banded zones (Fig. 11(b)) which is even higher than the blocking stress ( $-5 \mathrm{MPa}$ ). Moving a little bit across those defect lines, the magnitude of the mechanical field variables suddenly jumps to a different value. However, as the stress dependence in the reorientation strain, i.e., $\varepsilon^{\max }$ (see Table 6) is assumed constant in the current FE analysis, the blocking stress effect on strain could not be captured and we observe considerable amount reorientation strain at the highly stressed regions (Fig. 11(a)). 
Depending on situations, these unstable phenomena can have possible positive or negative impacts in the devices level applications. For example, in the current experimental conditions, a 2 [T] applied field may be considered as high. One way to reduce this value is to provide some boundary defects, such as small Vnotches, which will facilitate to nucleate and propagate more field singularities inside the materials and most of the interior regions will be filled up with the unstable zones. Such a phenomenon then increases the interior magnetic field intensities and facilitate variant reorientation at a low applied field. Note that singularities also increase the stress in the unstable zones and tries to suppress variant reorientation if the magnitude becomes more than the blocking stress. So, to obtain the best desired effects, one needs to optimize among the geometry with surface defects and the maximum/minimum field-stress requirements.

On the other hand, such an effect for Field Induced Phase Transfer (FIPT) (Haldar et al., 2014), where the blocking stress is much higher $(\approx-130 \mathrm{MPa}$ ) than variant reoriantation, may lead to a failure mechanism. The underlying mechanism in FIPT is phase transformation from anti-ferromagnetic martensite to the ferromagnetic autenite by applying magnetic field. Unfortunately, the applied field required for complete phase transformation is very high $(\approx 15[\mathrm{~T}])$. If we consider magnetic instability for such a material system, the stress in the banded regions could be large enough to cause a failure of the material. However, stability analysis of FIPT is not performed yet and we like to investigate the related issues in a future work.

\section{Conclusions}

We propose a magneto-mechanical coupled constitutive response for variant reorientation in single crystal MSMA. Discrete symmetry is taken into account to implement single crystal anisotropy. The developed constitutive equations are then considered in the coupled stability analysis. The analytic approach of stability analysis shows that the system becomes unstable during martensitic variant reorientation due to highly nonlinear magnetization constitutive response. We also have found that the single crystal anisotropy is an important factor and magneto-mechanical coupling due to anisotropy strongly influences the stability conditions. An analytic parametric study reveals that a material parameter which appears in the constitutive equations due to anisotropy could be responsible to make such a magneto-mechanically coupled material system unstable. FE analysis is performed to solve a coupled BVP. However, we only consider isotropy in the FE analysis as we aim to see the influence of magnetic instability on the mechanical field variables through coupling. The results show that the appearance of banded zones in the spacial distribution of the magnetic field variables as well as in the mechanical field variables, when loss of ellipticity occurs.

\section{Acknowledgments}

The authors would like to acknowledge the financial support of the Army Research Office, Grant no. W911NF-06-1-0319 for the initial stages of this work, NSF-IIMEC (International Institute for Multifunctional Materials for Energy Conversion) under Grant No. DMR-0844082, and NSF-NIRT, Grant no. CMMI: 0709283 for the support of the first author.

\section{Appendix}

Appendix A. Calculations for the system of 1st order PDEs

Eq. (41a) can be written as

$$
\begin{aligned}
& {\left[\begin{array}{ll}
1 & 0
\end{array}\right]\left(\begin{array}{l}
H_{1} \\
H_{2}
\end{array}\right)_{, 1}+\left[\begin{array}{ll}
0 & 1
\end{array}\right]\left(\begin{array}{l}
H_{1} \\
H_{2}
\end{array}\right)_{, 2}+\left[\begin{array}{ll}
1 & 0
\end{array}\right]\left(\begin{array}{l}
M_{1} \\
M_{2}
\end{array}\right)_{, 1}} \\
& +\left[\begin{array}{ll}
0 & 1
\end{array}\right]\left(\begin{array}{l}
M_{1} \\
M_{2}
\end{array}\right)_{, 2}=0,
\end{aligned}
$$

or

$$
\left[\begin{array}{ll}
1 & 0
\end{array}\right](\mathbf{H})_{, 1}+\left[\begin{array}{ll}
0 & 1
\end{array}\right](\mathbf{H})_{, 2}+\left[\begin{array}{ll}
1 & 0
\end{array}\right]\left[\mathbb{L}^{\prime}\right][\mathscr{E}](\mathbf{\Psi})_{, 1}+\left[\begin{array}{ll}
1 & 0
\end{array}\right]\left[\mathbb{K}^{\prime}\right](\mathbf{H})_{, 1}
$$$$
+\left[\begin{array}{ll}
0 & 1
\end{array}\right]\left[\mathbb{L}^{\prime}\right][\mathscr{E}](\mathbf{\Psi})_{, 2}+\left[\begin{array}{ll}
0 & 1
\end{array}\right]\left[\mathbb{R}^{\prime}\right](\mathbf{H})_{, 2}=0,
$$

or

$$
\left[\mathscr{H}_{1}\right](\mathbf{H})_{, 1}+\left[\mathscr{S}_{1}\right](\mathbf{\Psi})_{, 1}+\left[\mathscr{H}_{2}\right](\mathbf{H})_{, 2}+\left[\mathscr{S}_{2}\right](\mathbf{\Psi})_{, 2}=0 .
$$

Similarly, (41c) can be written as

$$
\begin{aligned}
& {\left[\begin{array}{lll}
1 & 0 & 0 \\
0 & 1 & 0
\end{array}\right]\left(\begin{array}{l}
\sigma_{11}^{E} \\
\sigma_{12}^{E} \\
\sigma_{22}^{E}
\end{array}\right)_{, 1}+\left[\begin{array}{lll}
0 & 1 & 0 \\
0 & 0 & 1
\end{array}\right]\left(\begin{array}{l}
\sigma_{11}^{E} \\
\sigma_{12}^{E} \\
\sigma_{22}^{E}
\end{array}\right)_{, 2}} \\
& +\mu_{0}\left[\begin{array}{ll}
H_{1} & 0 \\
H_{2} & 0
\end{array}\right]\left(\begin{array}{l}
H_{1} \\
H_{2}
\end{array}\right)_{, 1}+\mu_{0}\left[\begin{array}{ll}
0 & H_{1} \\
0 & H_{2}
\end{array}\right]\left(\begin{array}{l}
H_{1} \\
H_{2}
\end{array}\right)_{, 2}=0
\end{aligned}
$$

or

$$
\begin{aligned}
& {\left[\begin{array}{lll}
1 & 0 & 0 \\
0 & 1 & 0
\end{array}\right]\left(\boldsymbol{\sigma}^{E}\right)_{, 1}+\left[\begin{array}{lll}
0 & 1 & 0 \\
0 & 0 & 1
\end{array}\right]\left(\boldsymbol{\sigma}^{E}\right)_{, 2}+\mu_{0}\left[\begin{array}{ll}
H_{1} & 0 \\
H_{2} & 0
\end{array}\right](\mathbf{H})_{, 1}} \\
& +\mu_{0}\left[\begin{array}{ll}
0 & H_{1} \\
0 & H_{2}
\end{array}\right](\mathbf{H})_{, 2}=0,
\end{aligned}
$$

or

$$
\begin{aligned}
& {\left[\begin{array}{lll}
1 & 0 & 0 \\
0 & 1 & 0
\end{array}\right][\mathbb{L}][\mathscr{E}](\mathbf{\Psi})_{, 1}+\left[\begin{array}{lll}
1 & 0 & 0 \\
0 & 1 & 0
\end{array}\right][\mathbb{K}](\mathbf{H})_{, 1}+\left[\begin{array}{lll}
0 & 1 & 0 \\
0 & 0 & 1
\end{array}\right][\mathbb{L}][\mathscr{E}](\mathbf{\Psi})_{, 2}} \\
& +\left[\begin{array}{lll}
0 & 1 & 0 \\
0 & 0 & 1
\end{array}\right][\mathbb{K}](\mathbf{H})_{, 2}+\mu_{0}\left[\begin{array}{ll}
H_{1} & 0 \\
H_{2} & 0
\end{array}\right](\mathbf{H})_{, 1}+\mu_{0}\left[\begin{array}{ll}
0 & H_{1} \\
0 & H_{2}
\end{array}\right](\mathbf{H})_{, 2}=0,
\end{aligned}
$$

or we simply write

$$
\left[\mathscr{H}_{1}^{\prime}\right](\mathbf{H})_{, 1}+\left[\mathscr{S}_{1}^{\prime}\right](\mathbf{\Psi})_{, 1}+\left[\mathscr{H}_{2}^{\prime}\right](\mathbf{H})_{, 2}+\left[\mathscr{S}_{2}^{\prime}\right](\mathbf{\Psi})_{, 2}=0 .
$$

From (41b) we get

$H_{2,1}-H_{1,2}=0$,

and so

$$
\left[\begin{array}{ll}
0 & 1
\end{array}\right]\left(\begin{array}{l}
H_{1} \\
H_{2}
\end{array}\right)_{, 1}+\left[\begin{array}{ll}
-1 & 0
\end{array}\right]\left(\begin{array}{l}
H_{1} \\
H_{2}
\end{array}\right)_{, 2}=0
$$

or

$\left[\mathscr{C}_{1}\right](\mathbf{H})_{, 1}+\left[\mathscr{C}_{2}\right](\mathbf{H})_{, 2}=0$.

Finally, Eq. (41g), $\nabla \times \Psi=\epsilon_{i j k} \Psi_{m j},_{i} \mathbf{e}_{k} \otimes \mathbf{e}_{m}=0$, gives two additional equations 
$\Psi_{11,2}-\Psi_{12,1}=0$

$\Psi_{21,2}-\Psi_{22,1}=0$.

So we can write

$$
\left[\begin{array}{cccc}
0 & 0 & -1 & 0 \\
0 & -1 & 0 & 0
\end{array}\right]\left(\begin{array}{l}
\Psi_{11} \\
\Psi_{22} \\
\Psi_{12} \\
\Psi_{21}
\end{array}\right)_{, 1}+\left[\begin{array}{llll}
1 & 0 & 0 & 0 \\
0 & 0 & 0 & 1
\end{array}\right]\left(\begin{array}{l}
\Psi_{11} \\
\Psi_{22} \\
\Psi_{12} \\
\Psi_{21}
\end{array}\right)_{, 2}=0
$$

or

$$
\left[\mathscr{C}_{1}^{\prime}\right](\mathbf{\Psi})_{, 1}+\left[\mathscr{C}_{2}^{\prime}\right](\boldsymbol{\Psi})_{, 2}=0
$$

Combining (A-1), (A-2), (A-3) and (A-6) we write

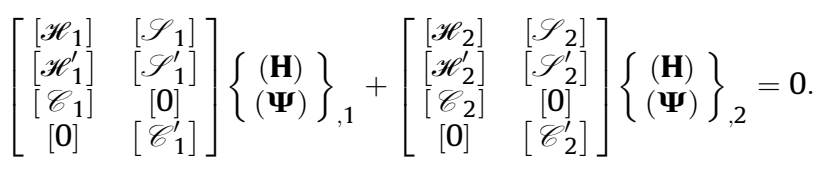

Appendix B. MSMA experiments and magneto-mechanical responses

In a typical experiment, the MSMA sample, initially in austenitic phase, is subjected to a constant compressive mechanical load along the $x$-axis to transform into stress induced variant (Fig. B.13). Then a magnetic field is applied along the perpendicular $y$-axis. After a critical value, field induced variant nucleates and both variants coexist by forming a twinned microstructure. Complete variant reorientation to field induced variant takes place at a high applied magnetic field.

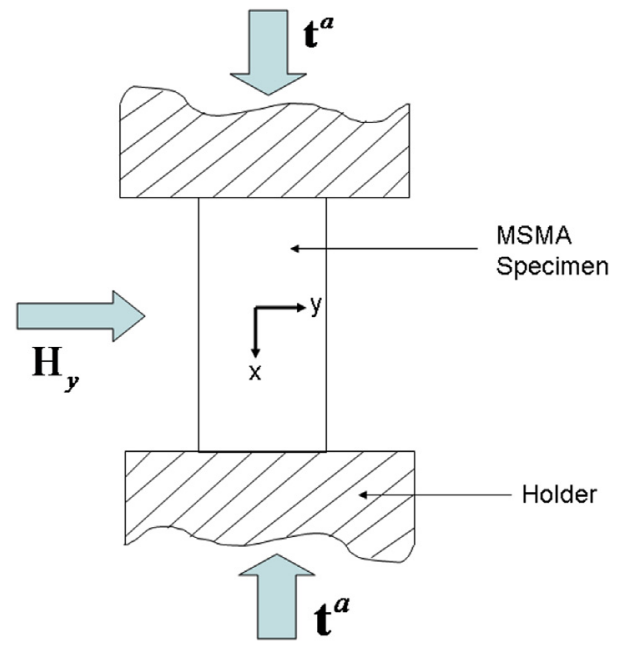

Fig. B.13. Schamatic of the experimental setup. Traction is applied in the vertical direction and magnetic field is applied in the horizontal direction.

As explained in Fig. B.14, we consider(A) an ideal stress favored variant with single magnetic domain in the initial configuration. $180^{\circ}$ domain walls in the variant- 1 is assumed to be eliminated by applying a small field along the easy axis.

When magnetic field is applied along the y-direction(hard axis), the magnetization vectors start rotating. Once the critical field for the variant reorientation has been reached, the field favored variant nucleates. In this configuration, $90^{\circ}$ domain forms due to the presence of twin variants (O'Handley et al., 2003; Tickle, 2000).

After complete reorientation, only field induced martensitic variant is present and the magnetization process becomes saturated. The magnetization vectors are aligned along the applied magnetic field, which is the easy axis of the field favored variant.

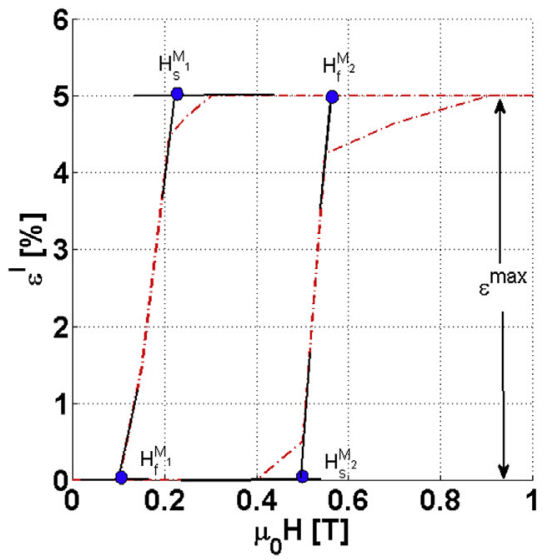

(a)

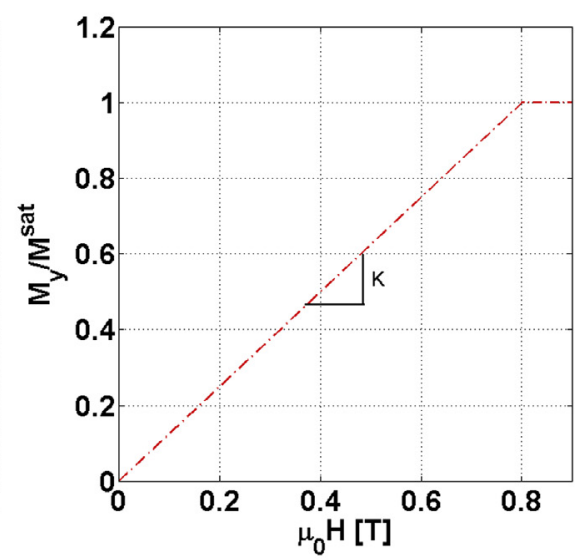

(b)

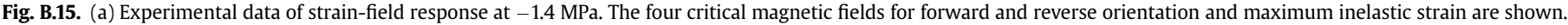
(b) Magnetization response of stress favored martensitic variant at a high stress level ( $-3 \mathrm{MPa}$ ), such that no reorientation takes place Heczko (2005). 
Under these circumstances, the $x$-component of the applied magnetic field is assumed to be zero. The magnetic field along the $x$ direction due to the magnetization of the body is assumed to be small and the dependence of $\boldsymbol{M}$ on $H_{1}$ is neglected. The magnetization components are assumed to have the form of $M_{1}=M_{1}\left(H_{2}\right)$ and $M_{2}=M_{2}\left(H_{2}\right)$. So, the components of the field variables are in the following form

$\boldsymbol{H}=\left\{0, H_{2}\right\}, \boldsymbol{M}=\left\{M_{1}, M_{2}\right\}$.

We also assume that the only non-zero stress component is $\sigma_{11}^{E}$, which is uniform and constant inside the specimen during the experiment.

\section{Appendix B.1. Material parameter identification}

We consider that the variant- 1 is under axial traction along the $x$ direction and magnetic field is applied along the $y$ direction, i.e, $\boldsymbol{\sigma}^{E}=\sigma_{11}^{E} \mathbf{i} \otimes \mathbf{i}$ and $\mathbf{H}=H_{2} \mathbf{j}$. Calibrations from different magnetomechanical parameters are given below.

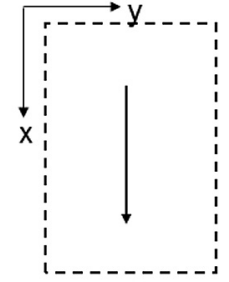

(A)

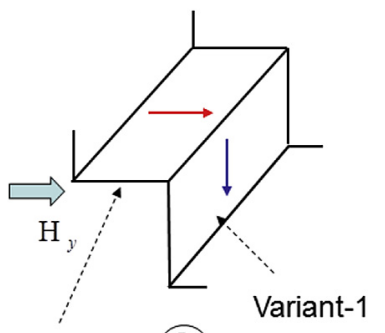

Variant-2 (C)

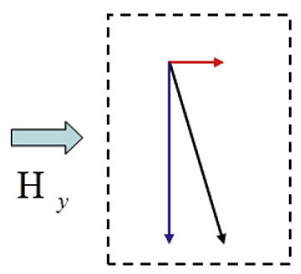

(B)

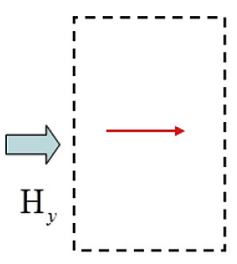

(D)
Fig. B.14. Schematic representation of micro scale mechanism.

\section{Appendix B.1.1. Mechanical response}

Due to lack of complete experimental data for the full anisotropic calibration of the model with the present magnetomechanical loading conditions, we only consider the x-component of the elastic and inelastic responses. No stress dependence on $\boldsymbol{\Lambda}^{r}$ is considered, i.e, $\boldsymbol{\Lambda}^{r}=d_{1} \mathbf{i} \otimes \mathbf{i}+d_{2} \mathbf{j} \otimes \mathbf{j}+d_{1} \mathbf{k} \otimes \mathbf{k}$ and so $\boldsymbol{\varepsilon}^{r}=\boldsymbol{\Lambda}^{r} \xi$. Then Eqs. (33)-(35) become

$\boldsymbol{\varepsilon}^{1}=2 a_{5} \sigma_{11}^{E} \mathbf{i} \otimes \mathbf{i}, \quad \boldsymbol{\varepsilon}^{2}=\left(2 b_{4} \sigma_{11}^{E}+b_{10} H_{2}\right) \mathbf{i} \otimes \mathbf{i}$,

$\boldsymbol{\varepsilon}^{I}=\tilde{d} \xi(\mathbf{i} \otimes \mathbf{i}-\mathbf{j} \otimes \mathbf{j})$.

The total number of unknown parameters are $a_{5}, b_{4}, b_{10}$, $\tilde{d}=\varepsilon_{11}^{r} t_{1}=d_{1} t_{1}$. We further assume $a_{5}=b_{4}=\frac{1}{2 \mathbb{E}}$, No magneto- elastic coupling, i.e, $b_{10}=0$. We set $t_{1} d_{1}=\varepsilon^{\text {max }}$, which can be found from Fig. B.15(a). Thus, the strain response can be reduced to

$\varepsilon_{11}=\left(\varepsilon_{1}\right)_{11}+\xi\left(\varepsilon_{2}-\varepsilon_{1}\right)_{11}+\left(\varepsilon^{I}\right)_{11}=\frac{1}{\mathbb{E}} \sigma_{11}^{E}+\varepsilon^{\max } \xi$.

Appendix B.1.2. Magnetic response

The expression of the magnetization can be written as

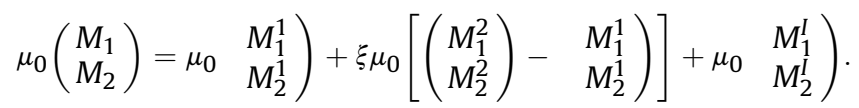

We assume no stress dependence in the magnetization of variant- 1 and variant-2, i.e, $a_{11}=b_{10}=0$ and Variant- 2 always be in saturation state, i.e, $b_{2}=0$. Then Eqs. (36)-(38) become

$\mu_{0} \mathbf{M}_{1}=a_{1} \mathbf{i}+2 a_{3} H_{2} \mathbf{j},, \quad \mu_{0} \mathbf{M}_{2}=b_{1} \mathbf{j}, \mu_{0} \mathbf{M}^{I}=p \xi \mathbf{j}$

and parameters to be identified are $a_{1}, a_{3}, b_{1}$ and $p=c_{5} d^{\prime}{ }_{1}$.

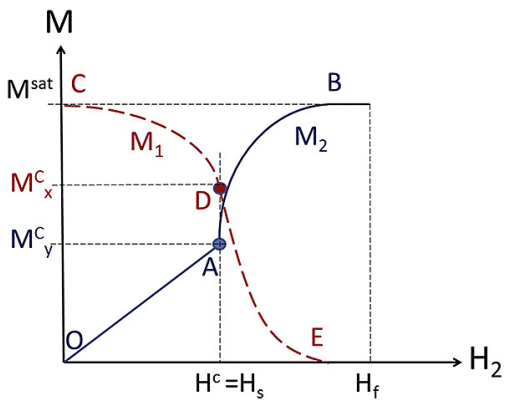

Fig. B.16. Schematic of magnetization response.

A typical experimental observation of the magnetization components is shown in Fig. B.16. $M_{y}^{C}$ is the $y$-component of the magnetization at the beginning of reorientation, i.e, at $H^{C}=H_{s}^{M_{2}}$. Knowing the experimental magnetization response (y-component) of a pure stress favored martensitic variant, as shown in Fig. B.15(b), we can immediately identify saturation magnetization $M^{\text {sat }}$ and the slope of the magnetization response $K$. Since $O A$ (Fig. B.16) is the linear response of the stress favored variant, $M_{y}^{C}$ can be calculated from $H_{s}^{M_{2}}$ and the slope $K$ as

$M_{y}^{C}=\mu_{0}\left(K M^{s a t}\right) H_{s}^{M_{2}}$.

We can write directly, from the y-component of (B-4)

$2 a_{3}=\frac{\mu_{0} M_{y}^{C}}{H^{C}}=\mu_{0} \beta$.

Knowing the parameters of $M_{2}$ (y-component) from the experiments, we will now determine the parameters pertaining to $M_{1}$ response if the micro-scale mechanism follows Fig. 14. Since the magnetization vectors are only rotating in the region $0<H_{2} \leq H^{C}$ (Fig. 14, Case-B), we always need to satisfy $\left|\mathbf{M}_{1}\right|=M^{\text {sat }}$, and so we can write 


$$
\begin{aligned}
& \left(M_{1}^{1}\right)^{2}+\left(M_{1}^{2}\right)^{2}=\left(M^{s a t}\right)^{2}, \\
& \Rightarrow a_{1}^{2}+4 a_{3}^{2} H_{2}^{2}=\left(\mu_{0} M^{s a t}\right)^{2}
\end{aligned}
$$

and then with the help of (B-6),

$a_{1}=\mu_{0} \sqrt{\left(M^{\text {sat }}\right)^{2}-\beta^{2} H_{2}^{2}}=\mu_{0} \tilde{a}_{1}$.

Note that $M_{x}^{C}$, the x-component of the magnetization at the beginning of reorientation $\left(H_{2}=H^{C}\right)$, can be written as

$M_{x}^{C}=\sqrt{\left(M^{s a t}\right)^{2}-\left(M_{y}^{C}\right)^{2}}$.

Since variant-2 is always saturated, from (B-4), $b_{1}=\mu_{0} M^{\text {sat }}$, which also implies $p^{\prime}=0$ as $\mu_{0} \mathbf{M}=\mu_{0} M^{\text {sat }} \mathbf{j}$ at $\xi=1$. Finally the magnetization response takes the following form:

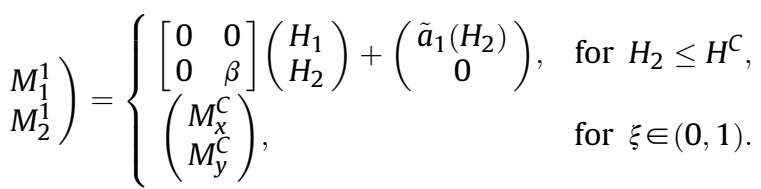

$$
\begin{aligned}
& \left.\begin{array}{l}
M_{1}^{2} \\
M_{2}^{2}
\end{array}\right)=\left(\begin{array}{c}
0 \\
M^{\text {sat }}
\end{array}\right), \\
& \left(\begin{array}{l}
M_{1}^{r} \\
M_{2}^{r}
\end{array}\right)=\left(\begin{array}{c}
0 \\
p^{\prime}
\end{array}\right) \xi .
\end{aligned}
$$

\section{Appendix B.1.3. Thermodynamic driving force}

The four critical magnetic fields are: the start of forward reorientation, $H_{s}^{M_{2}}$, the end of forward reorientation, $H_{f}^{M_{2}}$, the start of reverse reorientation, $H_{s}^{M_{1}}$, and the end of reverse reorientation, $H_{f}^{M_{1}}$ (Fig. B.15a). The reduced form of the thermodynamic force (6) is given by

$\pi^{r}=\sigma_{11}^{E} \varepsilon^{c u r}+\mu_{0} \Delta M_{2} H_{2}+f^{r}-\rho \Delta u_{0}$,

where $\rho \Delta u_{0}=\rho G_{0}^{2}-\rho G_{0}^{1}$. The hardening function is chosen as Lagoudas et al. (2011)



(a) $f^{r}:= \begin{cases}\frac{A}{2}\left(1+\xi^{n_{1}}-(1-\xi)^{n_{2}}\right)-B, & \dot{\xi}>0, \\ \frac{C}{2}\left(1+\xi^{n_{3}}-(1-\xi)^{n_{4}}\right)-D, & \dot{\xi}<0,\end{cases}$

where, $n_{1}, n_{2}, n_{3}$ and $n_{4}$ are some given exponents.

We need to know the parameters $A, B, C, D, Y^{r}$ and $\rho \Delta u_{0}$. From the Kuhn Tucker condition (8) we obtain two conditions at the beginning and two conditions at the finish of the forward reorientation. They are

$\pi^{r}\left(\sigma^{*}, H_{s}^{M_{2}}\right)-Y^{r}=0, \quad$ for $\dot{\xi}>0, \quad$ at $\xi=0$

$\pi^{r}\left(\sigma^{*}, H_{f}^{M_{2}}\right)-Y^{r}=0, \quad$ for $\dot{\xi}>0, \quad$ at $\xi=1$

Similarly, for reverse reorientation we get two more equations,

$$
\begin{aligned}
& \pi^{r}\left(\sigma^{*}, H_{s}^{M_{1}}\right)+Y^{r}=0, \quad \text { for } \dot{\xi}<0, \quad \text { at } \xi=1 \\
& \pi^{r}\left(\sigma^{*}, H_{f}^{M_{1}}\right)+Y^{r}=0, \quad \text { for } \dot{\xi}<0, \quad \text { at } \xi=0
\end{aligned}
$$

The constant stress level is denoted by $\sigma^{*}$. The continuity of the hardening function Lagoudas et al. (1996) gives us

$$
\left.\int_{0}^{1} f^{r}\right|_{\dot{\xi}>0} d \xi=\left.\int_{0}^{1} f^{r}\right|_{\xi<0} d \xi
$$

Solving the above five equations (from B-13a to B-15), we get the solution of five unknowns, $A, \tilde{B}, C, \tilde{D}, Y^{r}$. It should be noted that we introduce a new constant $\tilde{B}=B+\rho \Delta u_{0}$ and $\tilde{D}=D+\rho \Delta u_{0}$ since $B$ and $D$ absorbs the term $\rho \Delta u_{0}$. The detailed derivation can be found in the Appendix C. All the material constants are summarized in Table B.7.

\section{Table B.7}

Material constants.

$$
\begin{aligned}
& \mu_{0} H_{s}^{M_{2}}=0.5[\mathrm{~T}], \mu_{0} H_{f}^{M_{2}}=0.58[\mathrm{~T}], \mu_{0} H_{s}^{M_{1}}=0.28[\mathrm{~T}], \mu_{0} H_{f}^{M_{1}}=0.1[\mathrm{~T}] \\
& M^{\mathrm{s} a t}=742[\mathrm{kA} / \mathrm{m}], K^{\prime}=1.25[\mathrm{~T}]^{-1}, H^{\mathrm{C}}=H_{s}^{M_{2}}, \varepsilon^{\max }=5 \%, \mathbb{E}=2.0[\mathrm{GPa}] \\
& n_{1}=6.6, n_{2}=1.4, n_{3}=0.8, n_{4}=0.3, \sigma^{*}=-1.4[\mathrm{MPa}]
\end{aligned}
$$
$\mu_{0}=1.2566 \times 10^{-6}\left[\mathrm{~N} / \mathrm{A}^{2}\right]$

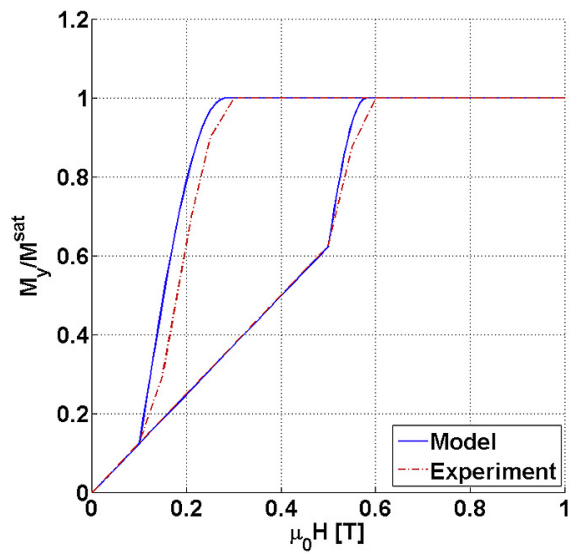

(b)

Fig. B.17. (a) Model simulation of strain-field response at $-1.4 \mathrm{MPa}$ and (b) model prediction of magnetization response at $-1.4 \mathrm{MPa}$. 
The model simulation is presented in Fig. B.17(a), followed by the magnetization prediction in Fig. B.17(b). We consider the following values for a transversely anisotropic medium (Table B.8) for the Sub-section 6.2.2: The values of $E_{1}=E, M_{x}^{C}$ and $\Delta M$ are taken from the isotropic material parameters, presented at Table B.7. The rest of the values are assumed to proceed for the numerical example.

\section{Table B.8}

Material parameters for anisotropic medium.

\begin{tabular}{llllll}
\hline$a_{5}$ & $a_{4}$ & $a_{12}$ & $a_{6}$ & $a_{1}$ & $b_{1}$ \\
\hline$\frac{1}{2 E_{1}}$ & $\frac{1}{2 E_{1}}$ & $-\frac{\nu_{12}}{E_{1}}=\frac{\nu_{21}}{E_{2}}$ & $G_{12}$ & $\mu_{0} M_{x}^{C}$ & $\mu_{0} \Delta M$ \\
$E_{1}[\mathrm{GPa}]$ & $E_{2}[\mathrm{GPa}]$ & $\nu_{12}$ & $\nu_{21}$ & $G_{12}[G P a]$ & \\
2 & 4 & 0.2 & 0.4 & 2.4 & \\
\hline
\end{tabular}

Appendix C. Calculations of hardening parameters

Combining the transformation function (7) with the driving force (B-11) and enforcing the Kuhn Tucker conditions (8), we can write the following conditions.

Appendix C.1. Forward transformation $(\dot{\xi}>0)$ :

$\pi^{r}\left(\sigma^{*}, H_{s}^{M_{2}}\right)-Y^{r}=0$ and at $\xi=0$

$\sigma^{*} \varepsilon^{\max }+\mu_{0} \Delta M H_{s}^{M_{2}}-\rho \Delta u_{0}-B-Y^{r}=0$,

$\pi^{r}\left(\sigma^{*}, H_{f}^{M_{2}}\right)-Y^{r}=0$ and at $\xi=1$

$\sigma^{*} \varepsilon^{\max }+\mu_{0} \Delta M H_{f}^{M_{2}}-\rho \Delta u_{0}+A-B-Y^{r}=0$.

Appendix C.2. Reverse transformation $(\dot{\xi}<0)$ :

$\pi^{r}\left(\sigma^{0 *}, H_{s}^{M_{1}}\right)+Y^{r}=0$ and at $\xi=1$

$\sigma^{*} \varepsilon^{\max }+\mu_{0} \Delta M H_{s}^{M_{1}}-\rho \Delta u_{0}+C-D+Y^{r}=0$,

$\pi^{r}\left(\sigma^{*}, H_{f}^{M_{1}}\right)+Y^{r}=0 \quad$ and at $\xi=0$

$\sigma^{*} \varepsilon^{\max }+\mu_{0} \Delta M H_{f}^{M_{1}}-\rho \Delta u_{0}-D+Y^{r}=0$,

\section{Appendix C.3. Continuity of Gibbs free energy potential}

The cyclic integral of the Gibbs free energy is zero. This implies that we need to satisfy the condition (B-15), i.e,

$\left.\int_{0}^{1} f^{r}\right|_{\dot{\xi}>0} d \xi=\left.\int_{0}^{1} f^{r}\right|_{\dot{\xi}<0} d \xi$

which gives us

$B-D=\frac{A}{2}\left[1+\frac{1}{n_{1}+1}-\frac{1}{n_{2}+1}\right]-\frac{C}{2}\left[1+\frac{1}{n_{3}+1}-\frac{1}{n_{4}+1}\right]$

So, from the five Eqs. ( $C-1)$ to $(C-5)$ we can now solve for five material parameters $A, B, C, D$ and $Y^{r}$. (C-1)-(C-2) gives

$A=\mu_{0} \Delta M\left(H_{s}^{M_{2}}-H_{f}^{M_{2}}\right)$,

(C-4)-(C-3) gives
$C=\mu_{0} \Delta M\left(H_{f}^{M_{1}}-H_{s}^{M_{1}}\right)$

$(\mathrm{C}-1)+(\mathrm{C}-4)$

$\begin{aligned} B+D & =2\left(\sigma^{*} \varepsilon^{\max }+\frac{\mu_{0}}{2} \Delta M\left(H_{s}^{M_{2}}+H_{f}^{M_{1}}\right)\right)-2 \rho \Delta u_{0} \\ & =2(\Theta)-2 \rho \Delta u_{0}\end{aligned}$

where, $\Theta$ denotes the expression under the braces. Solving (C-8) and $(\mathrm{C}-5)$ we get,

$\begin{aligned} B= & \Theta+\frac{A}{4}\left[1+\frac{1}{n_{1}+1}-\frac{1}{n_{2}+1}\right]-\frac{C}{4}\left[1+\frac{1}{n_{3}+1}-\frac{1}{n_{4}+1}\right] \\ & -\rho \Delta u_{0}\end{aligned}$

$D=\Theta-\frac{A}{4}\left[1+\frac{1}{n_{1}+1}-\frac{1}{n_{2}+1}\right]+\frac{C}{4}\left[1+\frac{1}{n_{3}+1}-\frac{1}{n_{4}+1}\right]$

$-\rho \Delta u_{0}$

and we denote

$$
\begin{aligned}
\tilde{B} & =B+\rho_{0} \Delta u_{0} \\
& =\Theta+\frac{A}{4}\left[1+\frac{1}{n_{1}+1}-\frac{1}{n_{2}+1}\right]-\frac{C}{4}\left[1+\frac{1}{n_{3}+1}-\frac{1}{n_{4}+1}\right] \\
\tilde{D} & =D+\rho_{0} \Delta u_{0} \\
& =\Theta-\frac{A}{4}\left[1+\frac{1}{n_{1}+1}-\frac{1}{n_{2}+1}\right]+\frac{C}{4}\left[1+\frac{1}{n_{3}+1}-\frac{1}{n_{4}+1}\right]
\end{aligned}
$$

Finally by (C-1) we get ${ }^{5}$

$Y^{r}=\sigma^{*} \varepsilon^{\max }+\mu_{0} \Delta M H_{s}^{M_{2}}-\tilde{B}$.

The evolution of the volume fraction for the forward reorientation is given below

Appendix C.4. Forward reorientation $(\dot{\xi}>0)$ :

$\Phi^{r} \dot{\xi}=0 \Rightarrow \Phi^{r}=0 \Rightarrow \pi^{r}-Y^{r}=0$

for which $\xi$ can be obtained by solving the following equation

$\sigma^{*} \varepsilon^{\max }+\mu_{0} \Delta M H_{2}+\frac{A}{2}\left(1+\xi^{n_{1}}-(1-\xi)^{n_{2}}\right)-\tilde{B}-Y^{r}=0$

Appendix C.5. Reverse reorientation $(\dot{\xi}<0)$ :

$\Phi^{r} \dot{\xi}=0 \Rightarrow \Phi^{r}=0 \Rightarrow \pi^{r}+Y^{r}=0$

for which $\xi$ can be obtained by solving the following equation

$\sigma^{*} \varepsilon^{\max }+\mu_{0} \Delta M H_{2}+\frac{C}{2}\left(1+\xi^{n_{3}}-(1-\xi)^{n_{4}}\right)-\tilde{D}+Y^{r}=0$

\footnotetext{
${ }^{5}$ For the anisotropic medium, as discussed in Subsection 6.2.2, due to the presence of constant $b_{10}$, we need to replace $\mu_{0} \Delta M$ by $\mu_{0} \Delta M+b_{10} \sigma^{*}$.
} 


\section{References}

Bradley, C.J., Cracknell, A.P., 1972. The Mathematical Theory of Symmetry in Solids. Oxford University Press.

Bustamante, R., Dorfmann, A., Ogden, R.W., 2008. On variational formulations in nonlinear magnetoelastostatics. Math. Mech. Solids 13, 725-745.

Coleman, B.D., Noll, W., 1963. The thermodynamics of elastic materials with heat conduction and viscosity. Arch. Ration. Mech. Anal. 13, 167-178.

Cracknell, A.P., 1975. Magnetism in Crystalline Materials. Pergamon Press.

DeSimone, A., 1993. Energy minimizers for large ferromagnetic bodies. Arch. Ration. Mech. Anal. 125, 99-143.

DeSimone, A., James, R.D., 1997. A theory of magnetostriction oriented towards applications. J. Appl. Phys. 81, 5706-5708.

DeSimone, A., James, R., 2002. A constrained theory of magnetoelasticity. J. Mech. Phys. Solids 50, 283-320.

DeSimone, A., James, R.D., 2003. Energetics of magnetoelastic domains in ferromagnetic shape memory alloys. J. de Phys. 112, 969-972.

DeSimone, A., Podio-Guidugli, P., 1996. On the continuum theory of deformable ferromagnetic solids. Arch. Ration. Mech. Anal. 136, 201-233.

Dorfmann, A., Ogden, R.W., 2004. Nonlinear magnetoelastic deformation. Q. J. Mech. Appl. Math. 57 (4), 599-622.

Dorfmann, A., Ogden, R.W., 2005. Nonlinear magnetoelastic deformations of elastomers. Acta Mech. 167, 13-28.

Ericksen, J.L., 2006. A modified theory of magnetic effects in elastic materials. Math. Mech. Solids 11, 23-47.

Eringen, A.C., Maugin, G.A., 1990. Electrodynamics of Continua I - Foundations and Solid Media. Springer-Verlag, New York.

Haldar, K., 2012. Magneto-thermo-mechanical Coupling. Stability Analysis an Phenomenological Constitutive Modeling of Magnetic Shape Memory Alloys. Ph.D. thesis. Texas A\&M University.

Haldar, K., Lagoudas, D.C., 2014. Constitutive modeling of magnetic shape memory alloys with discrete and continuous symmetries. Proc. R. Soc. Lond. Ser. A Math. Phys. Sci. 470, 20140216.

Haldar, K., Chatzigeorgiou, G., Lagoudas, D.C., 2010. Stability analysis of magnetostatic boundary value problems for magnetic smas. J. Intel. Mat. Syst. Struct. 21, $1103-1116$.

Haldar, K., Kiefer, B., Lagoudas, D.C., 2011. FE-analysis of the demagnetization effect and stress inhomogeneities in msma samples. Philos. Mag. 91 (32), 4126-4257.

Haldar, K., Lagoudas, D.C., Karaman, I., 2014. Magnetic field-induced martensitic phase transformation in magnetic shape memory alloys: modeling and experiments. J. Mech. Phys. Solids 69, 33-66.

Heczko, O., 2005. Magnetic shape memory effect and magnetization reversal. J. Magnet. Magnetic Mater. 290-291, 787-794.

Heczko, O., Straka, L., Ullakko, K., 2003. Relation between structure, magnetization process and magnetic shape memory effect of various martensites occurring in Ni-Mn-Ga alloys. J. de Phys. IV France 112, 959-962.

Hirsinger, L., Lexcellent, C., 2003a. Internal variable model for magneto-mechanical behaviour of ferromagnetic shape memory alloys Ni-Mn-Ga. J. de Phys. IV France 112, 977-980.

Hirsinger, L., Lexcellent, C., 2003b. Modelling detwinning of martensite platelets under magnetic and (or) stress actions on NiMnGa alloys. J. Magnet. Magnetic Mater. 254-255, 275-277.

Hutter, K, van de Ven, A.A.F. Ursescu, 2006. Electromagnetic Field Matter Interactions in Thermoelastic Solids and Viscous Fluids. Lecture Notes in Physics. Springer-Verlag, New York.

James, R.D., Wuttig, M., 1998. Magnetostriction of martensite. Philos. Mag. A 77, 1273-1299.

Kainuma, R., Imano, Y., Ito, W., Sutou, Y., Morito, H., Okamoto, S., Kitakami, O., Oikawa, K., Fujita1, A., Kanomata, T., Ishida, K., 2006. Magnetic-field-induced shape recovery by reverse phase transformation. Nature 439, 957-960.

Kankanala, S.V., Triantafyllidis, N., 2004. On finitely strained magnetorheological elastomers. J. Mech. Phys. Solids 52, 2869-2908.

Karaca, H.E., Karaman, I., Basaran, B., Chumlyakov, Y.I., Maier, H.J., 2006. Magnetic field and stress induced martensite reorientation in NiMnGa ferromagnetic shape memory alloy single crystals. Acta Mater. 54, 233-245.

Karaca, H.E., Karaman, I., Basaran, B., Lagoudas, D.C., Chumlyakov, Y.I., Maier, H.J., 2007. On the stress-assisted magnetic-field-induced phase transformation in $\mathrm{Ni}_{2} \mathrm{MnGa}$ ferromagnetic shape memory alloys. Acta Mater. 55, 4253-4269.

Karaca, H.E., Karaman, I., Basaran, B., Ren, Y., Chumlyakov, Y.I., Maier, H.J., 2009. Magnetic field-induced phase transformation in NiMnCoIn magnetic shape memory alloys-a new actuation mechanism with large work output. Adv. Funct. Mater. 19, 1-16.

Karaman, I., Basaran, B., Karaca, H.E., Karsilayan, A.I., Chumlyakov, Y.I., 2007. Energy harvesting using martensite variant reorientation mechanism in a NiMnGa magnetic shape memory alloy. Appl. Phys. Lett. 88, 172505.

Kiefer, B., Lagoudas, D.C., 2005. Magnetic field-induced martensitic variant reorientation in magnetic shape memory alloys. Phil. Mag. Special Issue Recent Adv. Theor. Mech. 85, 4289-4329 in Honor of SES 2003 A.C. Eringen Medalist G.A. Maugin.

Kiefer, B., Lagoudas, D.C., 2009. Modeling the coupled strain and magnetization response of magnetic shape memory alloys under magnetomechanical loading. J. Intel. Mat. Syst. Struct. 20, 143-170.
Kiefer, B., Karaca, H.E., Lagoudas, D.C., Karaman, I., 2006. Characterization and modeling of the magnetic field-induced strain and work output in $\mathrm{Ni}_{2} \mathrm{MnGa}$ shape memory alloys. J. Magnet. Magnetic Mater. 312, 164-175.

Lagoudas, D.C., Bo, Z., Qidwai, M.A., 1996. A unified thermodynamic constitutive model for SMA and finite element analysis of active metal matrix composites. Mech. Compos. Mater. Struct. 3, 153-179.

Lagoudas, D., Hartl, D., Chemisky, Y., Machado, L., Popov, P., 2011. Constitutive model for the numerical analysis of phase transformation in polycrystalline shape memory alloys. Int. J. Plast. http://dx.doi.org/10.1016/j.ijplas.2011.10.009.

Likhachev, A.A., Sozinov, A., Ullakko, K., 2004. Different modeling concepts of magnetic shape memory and their comparison with some experimental results obtained in Ni-Mn-Ga. Mat. Sci. Eng. A 378, 513-518.

McMeeking, R.M., Landis, C.M., 2005. Electrostatic forces and stored energy for deformable dielectric material. J. Appl. Mech. 72, 581-590.

McMeeking, R.M., Landis, C.M., Jimenez, S.M.A., 2007. A principle of virtual work for combined electrostatic and mechanical loading of materials. Int. J. Nonlinear Mech. 42, 831-838.

Miehe, C., Kiefer, B., Rosato, D., 2011a. An incremental variational formulation of dissipative magnetostriction at the macroscopic continuum level. Int. J. Solids Struct. 48, 1846-1866.

Miehe, C., Rosato, D., Kiefer, B., 2011b. Variational principles in dissipative electromagneto-mechanics: a framework for the macro-modeling of functional materials. Int. J. Numer. Methods Eng. 84, 1225-1276.

Müllner, P., Chernenko, V.A., Kostorz, G., 2003. A microscopic approach to the magnetic-field-induced deformation of martensite (magnetoplasticity). J. Magnet. Magnetic Mater. 267, 325-334.

Murray, S.J., Marioni, M., Tello, P.G., Allen, S.M., O'Handley, R.C., 2001a. Giant magnetic-field-induced strain in Ni-Mn-Ga crystals: experimental results and modeling. J. Magnet. Magnetic Mater. 226-230, 945-947.

Murray, S.J., O'Handley, R.C., Allen, S.M., 2001b. Model for discontinuous actuation of ferromagnetic shape memory alloy under stress. J. Appl. Phys. 89, 1295-1301.

O'Handley, R.C., 1998. Model for strain and magnetization in magnetic shapememory alloys. J. Appl. Phys. 83, 3263-3270.

O'Handley, R.C., Murray, S.J., Marioni, M., Nembach, H., Allen, S.M., 2000. Phenomenology of giant magnetic-field-induced strain in ferromagnetic shapememory materials (invited). J. Appl. Phys. 87, 4712-4717.

O'Handley, R.C., Allen, S.M., Paul, D.I., Henry, C.P., Marioni, M., Bono, D., Jenkins, C., Banful, A., Wager, R., 2003. Keynote address: magnetic field-induced strain in single crystal Ni-Mn-Ga. In: Proceedings of SPIE, Symposium on Smart Structures and Materials 5053, pp. 200-206.

Pasquale, M., 2003. Mechanical sensors and actuators. Sensors Actuat. A 106 $142-148$.

Penfield, P.J., Haus, H.A., 1967. Electrodynamics of Moving Media. The MIT Press, Cambridge, Massachusetts.

Podio-Guidugli, P., Roubicek, T., Tomassetti, G., 2010. A thermodynamically consistent theory of the ferro/paramagnetic transition. Arch. Ration. Mech. Anal. 198, 1057-1094.

Qidwai, M.A., Lagoudas, D.C., 2000. Numerical impementation of a shape memory alloy thermomechanical constitutive model using return mapping algorithms. Int. J. Numer. Methods Eng. 47, 1123-1168.

Sarawate, N., Dapino, M., 2006. Experimental characterization of the sensor effect in ferromagnetic shape memory NiMnGa. Appl. Phys. Lett. 88, 121923.

Sarawate, N.N., Dapino, M.J., 2007. A continuum thermodynamics model for the sensing effect in ferromagnetic shape memory ni-mn-ga. J. Appl. Phys. 101, 123522.

Shield, T.W., 2003. Magnetomechanical testing machine for ferromagnetic shapememory alloys. Rev. Sci. Instrum. 74, 4077-4088.

Simo, J.C., Hughes, T.J.R., 1998. Computational Inelasticity. In: volume 7 of Interdisciplinary Applied Mathematics. Springer-Verlag, New York.

Steigmann, D.J., 2004. Equilibrium theory for magnetic elastomers and magnetoelastic membranes. Non-Linear Mech. 39, 1193-1216.

Steigmann, D.J., 2009. On the formulation of balance laws for electromagnetic continua. Math. Mech. Solids 14, 390-402.

Sutoua, Y., Imano, Y., Koeda, N., Omori, T., Kainuma, R., Ishidab, K., Oikawab, K., 2004. Magnetic and martensitic transformations of $\mathrm{NiMnX}$ ( $\mathrm{X}=\mathrm{In}, \mathrm{Sn}, \mathrm{Sb})$ ferromagnetic shape memory alloys. Appl. Phys. Lett. 85, 4358-4360.

Tellinen, J., Suorsa, I., Jääskeläinen, Aaltio, I., Ullakko, K., 2002. Basic properties of magnetic shape memory actuators. In: Proceedings of the 8th International Conference ACTUATOR 2002, Bremen, Germany, 10-12 June 2002, pp. 566-569.

Tickle, R., 2000. Ferromagnetic Shape Memory Materials. Ph.D. thesis. University of Minnesota, Minneapolis, MN.

Toupin, R., 1956. The elastic dielectric. J. Ration. Mech. Anal. 5, 849-915.

Toupin, R., 1960. Stress tensors in elastic dielectrics. Arch. Ration. Mech. Anal. 5, 440-452.

Wang, J., Steinmann, P., 2012. A variational approach towards the modeling of magnetic field induced strains in magnetic shape memory alloys. J. Mech. Phys. Solids 60, 1179.

Yamamoto, T., Taya, M., Sutou, Y., Liang, Y., Wada, T., Sorensen, L., 2004. Magnetic field-induced reversible variant rearrangement in Fe-Pd single crystals. Acta Mater. 52, 5083-5091. 